\title{
GENERAL TREATMENT OF VORTICAL, TOROIDAL, AND COMPRESSION MODES
}

\author{
J. Kvasil ${ }^{1}$, V.O. Nesterenko ${ }^{2}$, W. Kleinig ${ }^{2,3}$, P.-G. Reinhard ${ }^{4}$, and P. Vesely ${ }^{1,5}$ \\ 1 Institute of Particle and Nuclear Physics, Charles University, CZ-18000, Praha 8, Czech Republi泮 \\ ${ }^{2}$ Laboratory of Theoretical Physics, Joint Institute for Nuclear Research, Dubna, Moscow region, 141980, Russid \\ 3 Technische Universität Dresden, Inst. für Analysis,D-01062, Dresden, Germany \\ 4 Institut für Theoretische Physik II, Universität Erlangen, D-91058, Erlangen, Germany and \\ 5 Department of Physics, P.O. Box 35 (YFL) FI-40014, University of Jyväskylä, Finland
}

(Dated: November 13, 2018)

\begin{abstract}
The multipole vortical, toroidal, and compression modes are analyzed. Following the vorticity concept of Ravenhall and Wambach, the vortical operator is derived and related in a simple way to the toroidal and compression operators. The strength functions and velocity fields of the modes are analyzed in ${ }^{208} \mathrm{~Pb}$ within the random-phase-approximation using the Skyrme force SLy6. Both convection and magnetization nuclear currents are taken into account. It is shown that the isoscalar (isovector) vortical and toroidal modes are dominated by the convection (magnetization) nuclear current while the compression mode is fully convective. The relation between the above concept of the vorticity to the hydrodynamical vorticity is briefly discussed.
\end{abstract}

\section{INTRODUCTION}

An irrotational character of nuclear flow is a basic assumption in collective nuclear dynamics [1] 4], which manifests itself in numerous examples of low-energy excitations and giant resonances (GR). At the same time, nuclear motion can also carry a vorticity, i.e. a deviation from irrotational flow [5 8]. In hydrodynamics (HD), the vorticity is defined as a curl of the velocity field [9]. Instead, the nuclear theory deals with nuclear currents rather than velocities and so here the vorticity is often defined through the $j_{\lambda \lambda+1}(r)$ component of the multipole decomposition of the transverse nuclear current [7]. The component $j_{\lambda \lambda+1}(r)$ is treated as unrestricted by the continuity equation (containing the current divergence $\vec{\nabla} \cdot \vec{j}$ ) and so is believed to be of a vortical character. In this case, the vorticity and charge transition density represent two independent parts of the charge-current distribution. This consideration reminds the previous result [10] where the current component $j_{\lambda \lambda-1}(r)$ is proposed to be constrained by the continuity equation and thus determined by the charge distribution while the component $j_{\lambda \lambda+1}(r)$ is treated as independent.

Both definitions of the vorticity, from HD and Ref. [7], are widely used in the literature: the former in the nuclear fluid-dynamical models (see, e.g. [11] and references therein) and the latter in the microscopic studies, see e.g. [7, 8]. These two definitions are assumed to be closely related [7], though they are different observables by construction. Actually, they represent different aspects of the nuclear vorticity. In this paper, we will concentrate on the $j_{\lambda \lambda+1}(r)$-based vorticity [7]. The comparison with the HD case will be also done.

The most remarkable manifestation of vorticity is the

\footnotetext{
*Electronic address: kvasil@ipnp.troja.mff.cuni.cz
}

${ }^{\dagger}$ Electronic address: nester@theor.jinr.ru electric dipole toroidal mode (TM) [12-14] intensively explored during the last decades, see e.g. the review [15] and references therein. This exotic mode is treated as a vortical collective motion of the toroidal type. The TM operator is the second-order correction to the leading E1 operator in the long-wave approximation. Another kind of the second-order E1 flow is represented by the anisotropic compression mode (CM), often called the isoscalar dipole GR [16 19]. The mode is viewed as a compression wave in a definite direction and so is related to the nuclear incompressibility [16, 19]. The isoscalar $(\mathrm{T}=0) \mathrm{TM}$ and $\mathrm{CM}$ were observed in $\left(\alpha, \alpha^{\prime}\right)$-reaction as broad low-energy (TM dominated) and high-energy (CM dominated) electric dipole distributions [14, 18, 20 25]. The TM was also investigated in the region of the pygmy resonance in ${ }^{208} \mathrm{~Pb}$ in a nuclear fluorescence experiment 26. Perhaps, the TM can be discriminated in the transverse $\left(e, e^{\prime}\right)$ form-factors [27].

The TM and CM were examined in various models, including the fluid-dynamical and sum-rule approaches [11, 13, 19, 28], the method of Wigner function moments [29, 30], the random-phase-approximation (RPA) [31] and multi-phonon [26] methods with phenomenological single-particle potentials. More refined RPA studies within the self-consistent mean-field approaches were also performed, relativistic ones [32, 33] and those based on Skyrme forces [34, 35], for a review see [15]. A direct relation between the current-dependent TM and densitydependent CM operators was established in [13, 31] and both modes were shown to be mixed. Most of the studies reproduce the observed bimodal (low-energy TM and high-energy CM) distribution. However, theoretical models generally overestimate the CM peak energy by $\sim 4 \mathrm{MeV}$ and underestimates the TM one by 1-2 MeV. Besides, they yield a much broader TM distribution [15].

A special effort was devoted to the nuclear vorticity as such [5 8]. The possibility to measure the vorticity in $\left(e, e^{\prime}\right)$ experiments was discussed [8, 27, 36].

Despite these thorough studies, some principle points 
concerning the vorticity and related modes deserve further inspection: i) There is an essential difference in modeling the vortical mode (VM) and their TM and CM counterparts. The TM and CM are usually deduced as second order terms in a low-momentum expansion of the electric multipole transition operators [12, 13]. To the best of our knowledge, an analogous way to the VM operator has yet to be developed. This would help to establish a formal relation between VM and TM/CM. ii) Most of the previous studies (with exception of [7, 8, 31] ) employ only the convection part $j_{c}$ of the nuclear current and skip its magnetization (spin) part $j_{m}$, though the latter can also produce a vorticity. The role of $j_{m}$ in VM and other modes has yet to be clarified. iii) Mainly the $\mathrm{T}=0$ channel of the modes were discussed, although their $\mathrm{T}=1$ counterpart is also interesting and deserves a closer look. iv) The relation between two definition of the vorticity, from HD [9] and $j_{\lambda \lambda+1}(r)$ current component [7], has yet to be clarified.

The aim of the present study is to explore the open problems listed above. First of all, the vortical operator unconstrained by the charge conservation is derived following the ideas [7]. The operator has a simple relation with its TM/CM counterparts and is also deduced as a second-order term in a low-momentum (long wavelength) expansion of the dominant electric operator. Further, the difference in the vorticity criteria from the HD 9] and Ref. [7] is inspected. In the numerical calculations, both $\mathrm{T}=0$ and $\mathrm{T}=1$ channels of VM, TM, and CM are analyzed by using the full nuclear current $j=j_{c}+j_{m}$. The dominant role of $j_{m}$ and thus the spin vorticity in forming the isovector VM and TM is worked out. Both single-particle and collective mechanisms of the vorticity are discussed.

The numerical analysis is done within the selfconsistent separable random-phase-approximation (SRPA) approach based on the factorized Skyrme residual interaction [37, 38. The systematic studies of electric [37 42] and magnetic [43 45] GR in spherical and deformed nuclei have established this approach as a reliable and effective theoretical tool.

The paper is organized as follows. In Sec. II, the nuclear vorticity is discussed in context of the previous studies. In Sec. III, the VM, TM, and CM operators are derived on the same theoretical grounds, following the prescription [7]. A simple relation between the operators is established. The mode velocities are analyzed and different criteria of the vorticity, from [7] and HD, are compared. Sec. IV provides an outline of the calculation scheme within the Skyrme SRPA. In Sec. V, the numerical results for the $\mathrm{T}=0$ and $\mathrm{T}=1 \mathrm{VM}, \mathrm{TM}$, and $\mathrm{CM}$ are discussed. A summary is given in Sec. VI. Appendix A justifies the procedure of building the vortical operator. Appendix $\mathrm{B}$ describes the procedure for extraction of the spurious center-of-mass corrections. Appendix C provides the explicit expressions for the density and current operators. Appendix D sketches the basics of SRPA.

\section{VORTICITY IN TERMS OF VELOCITIES AND CURRENTS}

The HD nuclear models, including the famous liquiddrop model, assume an irrotational character of the collective nuclear flow [1 3 ]

$$
\vec{\nabla} \times \vec{v}(\vec{r})=0
$$

where $\vec{v}(\vec{r})$ is the local velocity field. The deviation from the irrotational flow is quantified by the HD vorticity

$$
\vec{\varpi}(\vec{r})=\vec{\nabla} \times \vec{v}(\vec{r})
$$

Unlike the HD models, nuclear theory prefers to deal with currents $\vec{j}_{n u c}(\vec{r})$ rather than velocities $\vec{v}(\vec{r})$. However, Eqs. (1) and (2) cannot be replaced by the similar expressions for the nuclear current since, as shown below, the curls of velocity and current have a different structure. Moreover, $\vec{\nabla} \times \vec{j}_{n u c}(\vec{r})$ is the key part of the electrical multipole operator $\hat{M}(E \lambda \mu, k)$ and, so, treating $\vec{\nabla} \times \vec{j}_{n u c}(\vec{r})$ as a vortical quantity would wrongly mean a fully vortical nature of any $E \lambda \mu$ excitations, which contradicts, e.g., a predominantly irrotational character of electric GR.

For using the HD definition of vorticity (2), the quantum theory should express it through the nuclear current. A common way is to define the velocity field $\vec{v}_{\nu}(\vec{r})$ for the excitation mode $\nu$ through the current transition density $\delta \vec{j}_{\nu}(\vec{r})$ and ground state density $\rho_{0}(\vec{r})[6,46]$,

$$
\delta \vec{j}_{\nu}(\vec{r})=\rho_{0}(\vec{r}) \vec{v}_{\nu}(\vec{r})
$$

which casts (2) into the form

$$
\vec{\nabla} \times \vec{v}_{\nu}(\vec{r})=\frac{\rho_{0}(\vec{r}) \vec{\nabla} \times \delta \vec{j}_{\nu}(\vec{r})-\vec{\nabla} \rho_{0}(\vec{r}) \times \delta \vec{j}_{\nu}(\vec{r})}{\rho_{0}^{2}(\vec{r})} .
$$

This illuminates the difference between curls of the velocity and current and thus shows that $\vec{\nabla} \times \delta \overrightarrow{j_{\nu}}(\vec{r})$ cannot be a measure of vorticity. The difference is comprised in the gradient field $\vec{\nabla} \rho_{0}(\vec{r})$ which is maximal at the nuclear surface and minimal in the interior.

In [7], a concept of nuclear vorticity, alternative to the HD one and fully based on the nuclear current, was proposed. It aims to find a component of the nuclear current which is not restricted by the continuity equation

$$
\vec{\nabla} \cdot \delta \vec{j}_{\nu}(\vec{r})=-i k c \delta \rho_{\nu}(\vec{r})
$$

with $k$ being the transfer momentum. By construction, this current component should not contribute to the irrotational motion and vanish in the divergence $\vec{\nabla} \cdot \delta \overrightarrow{j_{\nu}}(\vec{r})$. So it may be naturally used for building the vortical quantities.

Since the present study follows similar lines, it is worth to outline the concept [7] in more detail. In spherical 
nuclei, it exploits the multipole expansion of the nucleon and current transition densities:

$$
\begin{aligned}
\delta \rho_{f i}(\vec{r}) & =\left\langle j_{f} m_{f}|\hat{\rho}(\vec{r})| j_{i} m_{i}\right\rangle \\
& =\sum_{\lambda \mu} a_{\lambda \mu}^{f i} \rho_{\lambda}(r) Y_{\lambda \mu}(\hat{\vec{r}}), \\
\delta \vec{j}_{f i}(\vec{r}) & =\left\langle j_{f} m_{f}\left|\hat{\vec{j}}_{\mathrm{n} u c}(\vec{r})\right| j_{i} m_{i}\right\rangle \\
& =-i \sum_{\lambda \mu} \sum_{L=\lambda \pm 1} a_{\lambda \mu}^{f i} j_{\lambda L}(r) \vec{Y}_{\lambda L \mu}(\hat{\vec{r}}),
\end{aligned}
$$

where

$$
a_{\lambda \mu}^{f i}=(-1)^{\mu} \frac{\left(j_{i} m_{i} \lambda-\mu \mid j_{f} m_{f}\right)}{\sqrt{2 j_{f}+1}},
$$

$j_{i}, m_{i}\left(j_{f}, m_{f}\right)$ are spins and their projections for the initial $i$ (final $f$ ) state, $Y_{\lambda \mu}(\hat{\vec{r}})$ and $\vec{Y}_{\lambda L \mu}(\hat{\vec{r}})$ are ordinary and vector spherical harmonics [47]. The $\rho_{\lambda}(r)$ and $j_{\lambda L}(r)$ are multipole components of the transition density and current. Using the above expansions and the quantity

$$
\delta \vec{S}_{f i}(\vec{r})=\sum_{\lambda \mu} a_{\lambda \mu}^{f i} \sqrt{\frac{\lambda+1}{\lambda}} k c \rho_{\lambda}(r) \vec{Y}_{\lambda \lambda \mu}(\hat{\vec{r}}),
$$

the unconstrained vortical transition density

$$
\begin{aligned}
\delta \vec{w}_{f i}(\vec{r}) & =\vec{\nabla} \times \delta \vec{j}_{f i}(\vec{r})-\delta \vec{S}_{f i}(\vec{r}) \\
& =\sum_{\lambda \mu} a_{\lambda \mu}^{f i} w_{\lambda \lambda}(r) \vec{Y}_{\lambda \lambda \mu}(\hat{\vec{r}})
\end{aligned}
$$

is built [7], where the vortical multipoles

$$
w_{\lambda \lambda}(r)=\sqrt{\frac{2 \lambda+1}{\lambda}}\left(\frac{d}{d r}+\frac{\lambda+2}{r}\right) j_{\lambda \lambda+1}(r)
$$

are determined by the radial current component $j_{\lambda \lambda+1}(r)$. Finally, the vorticity strength is given by

$$
\nu_{\lambda}=\int_{0}^{\infty} d r r^{\lambda+4} w_{\lambda \lambda}(r) .
$$

The continuity equation (5) in terms of the $r^{\lambda}$ moments relates the current component $j_{\lambda \lambda-1}(r)$ to the transition density but leaves the component $j_{\lambda \lambda+1}(r)$ untouched 7]. So, just $j_{\lambda \lambda-1}(r)$ provides the charge-conservation constraint and the quantity (9) is constructed so as to remove $j_{\lambda \lambda-1}(r)$ from $\vec{\nabla} \times \delta \vec{j}_{f i}(\vec{r})$, see more discussion in Appendix $\mathrm{A}$. The unconstrained vortical value (11) includes only $j_{\lambda \lambda+1}(r)$. Moreover, following [7], the motion is treated as vortical if its current involves $j_{\lambda \lambda+1}(r)$.

The formalism [7] treats the vorticity without using an explicit vortical operator analogous to the TM and $\mathrm{CM}$ ones. However, we need such operator for reasons of comparison of vortical modes with TM and CM. In the next section, we will develop the explicit vortical operator by using the prescription [7] and relate this operator with the toroidal and compression ones. The subsequent discussion will show that this operator is not truly vortical in the HD sense and the presence of $j_{\lambda \lambda+1}(r)$ in the current is not enough for the HD vorticity.

\section{VM, TM, AND CM OPERATORS}

\section{A. Derivation of VM, TM and, CM operators}

The standard electrical multipole operator may be written in different forms [48]:

$$
\begin{aligned}
\hat{M}(E \lambda \mu, k)= & -i \frac{(2 \lambda+1) ! !}{c k^{\lambda+1}(\lambda+1)} \\
& \cdot \int d^{3} r \hat{\vec{j}}_{\mathrm{n} u c}(\vec{r}) \cdot\left[\vec{\nabla} \times(\vec{r} \times \vec{\nabla}) j_{\lambda}(k r) Y_{\lambda \mu}(\hat{\vec{r}})\right] \\
= & \frac{(2 \lambda+1) ! !}{c k^{\lambda+1}} \sqrt{\frac{\lambda}{\lambda+1}} \\
& \cdot \int d^{3} r\left[j_{\lambda}(k r) \vec{Y}_{\lambda \lambda \mu}(\hat{\vec{r}})\right] \cdot\left[\vec{\nabla} \times \hat{\vec{j}}_{\mathrm{n} u c}(\vec{r})\right]
\end{aligned}
$$

where $j_{\lambda}(k r)$ is the spherical Bessel function.

The form (14) shows that $\vec{\nabla} \times \hat{\vec{j}}_{n u c}(\vec{r})$ cannot be a measure of the vorticity since otherwise $\hat{M}(E \lambda \mu, k)$ would indicate only vortical electric excitations. At the same time, the form (14) suggests that the vortical operator may be built from $\hat{M}(E k \lambda \mu)$ by replacing $\vec{\nabla} \times \hat{\vec{j}}_{\text {nuc }}(\vec{r})$ with the truly vortical quantity [7]

$$
\vec{\nabla} \times \hat{\vec{j}}_{\mathrm{n} u c}(\vec{r})-\frac{i}{\lambda} k c[\vec{\nabla} \hat{\rho}(\vec{r}) \times \vec{r}]
$$

The density-dependent term in (15) subtracts the charge conservation constraint. Actually it plays a similar role as the r.h.s. second term in the vortical transition density (10). Both prescriptions, (10) and (15), have the same intention but, being applied to to different quantities (transition densities and operators), lead to formally different recipes. While (10) excludes the $j_{\lambda \lambda-1}(r)$ terms, the recipe (15) gives an exact compensation of the lowest-order $k$-terms in the final vortical operator, see the derivation below. Both (10) and (15) remind the r.h.s. of the HD expression (41). They are compared and discussed in more detail in Appendix A

By using (15), the vortical operator is defined as

$$
\hat{M}_{\mathrm{vor}}(E \lambda \mu, k)=\hat{M}(E \lambda \mu, k)-\hat{M}_{S}(E \lambda \mu, k)
$$

i.e. as a difference of the electric operator (14) and the subsidiary operator

$$
\begin{aligned}
\hat{M}_{S}(E \lambda \mu, k)= & i \frac{(2 \lambda+1) ! !}{k^{\lambda} \sqrt{\lambda(\lambda+1)}} \\
& \cdot \int d^{3} r\left[j_{\lambda}(k r) \vec{Y}_{\lambda \lambda \mu}(\hat{\vec{r}})\right] \cdot[\vec{\nabla} \hat{\rho}(\vec{r}) \times \vec{r}] .
\end{aligned}
$$


The latter may be also written in the forms

$$
\begin{aligned}
& \hat{M}_{S}(E \lambda \mu, k)= \\
= & -\frac{(2 \lambda+1) ! !}{k^{\lambda} \sqrt{\lambda(\lambda+1)}} \int d^{3} r \hat{\rho}(\vec{r}) j_{\lambda}(k r) \hat{\vec{l}} \cdot \vec{Y}_{\lambda \lambda \mu}(\hat{\vec{r}}) \\
= & -\frac{(2 \lambda+1) ! !}{k^{\lambda}} \int d^{3} r \hat{\rho}(\vec{r}) j_{\lambda}(k r) Y_{\lambda \mu}(\hat{\vec{r}}) \\
= & -i \frac{(2 \lambda+1) ! !}{c k^{\lambda+1}} \int d^{3} r j_{\lambda}(k r) Y_{\lambda \mu}(\hat{\vec{r}})\left[\vec{\nabla} \cdot \hat{\vec{j}}_{\mathrm{n} u c}(\vec{r})\right]
\end{aligned}
$$

The form (20) is obtained by using the operator continuity equation

$$
\vec{\nabla} \cdot \hat{\vec{j}}_{\mathrm{n} u c}=-\frac{i}{\hbar}[\hat{H}, \hat{\rho}]=-i k c \hat{\rho} .
$$

In the long-wavelength approximation $(k \rightarrow 0)$, we keep only the first and second terms in the expansion of the spherical Bessel function

$$
j_{\lambda}(k r)=\frac{(k r)^{\lambda}}{(2 \lambda+1) ! !}\left[1-\frac{(k r)^{2}}{2(2 \lambda+3)}+\ldots\right]
$$

and thus get for the electric and subsidiary operators

$$
\begin{aligned}
\hat{M}(E \lambda \mu, k) & \approx \hat{M}(E \lambda \mu)+k \hat{M}_{\mathrm{tor}}(E \lambda \mu), \\
\hat{M}_{S}(E \lambda \mu, k) & \approx \hat{M}(E \lambda \mu)-k \hat{M}_{\mathrm{com}}(E \lambda \mu),
\end{aligned}
$$

where

$$
\begin{aligned}
\hat{M}(E \lambda \mu) & =\frac{i}{k c} \int d^{3} r \hat{\vec{j}}_{\mathrm{n} u c}(\vec{r}) \cdot \vec{\nabla}\left(r^{\lambda} Y_{\lambda \mu}(\hat{\vec{r}})\right) \\
& =-\frac{i}{k c} \int d^{3} r\left(\vec{\nabla} \cdot \hat{\vec{j}}_{\mathrm{n} u c}(\vec{r})\right) r^{\lambda} Y_{\lambda \mu}(\hat{\vec{r}}) \\
& =-\int d^{3} r \hat{\rho}(\vec{r}) r^{\lambda} Y_{\lambda \mu}(\hat{\vec{r}})
\end{aligned}
$$

Using the definition, $\hat{M}_{\mathrm{vor}}(E \lambda \mu, k)=k \hat{M}_{\mathrm{vor}}(E \lambda \mu)$, we finally come to the relation

$$
\hat{M}_{\mathrm{vor}}(E \lambda \mu)=\hat{M}_{\mathrm{tor}}(E \lambda \mu)+\hat{M}_{\mathrm{com}}(E \lambda \mu),
$$

where is the familiar electrical operator in the long-wavelength limit (lowest order term) and

$$
\begin{aligned}
\hat{M}_{\mathrm{tor}}(E \lambda \mu)= & \frac{i}{2 c(\lambda+1)(2 \lambda+3)} \\
& \cdot \int d^{3} r \hat{\vec{j}}_{\mathrm{n} u c}(\vec{r}) \cdot\left[\vec{\nabla} \times(\vec{r} \times \vec{\nabla}) r^{\lambda+2} Y_{\lambda \mu}(\hat{\vec{r}})\right] \\
= & \frac{i}{c(\lambda+1)} \int d^{3} r \hat{\vec{j}}_{\mathrm{n} u c}(\vec{r}) \cdot \vec{r} r^{\lambda} Y_{\lambda \mu}(\hat{\vec{r}}) \\
+ & k \frac{\lambda+3}{2(\lambda+1)(2 \lambda+3)} \int d^{3} r \hat{\rho}(\vec{r}) r^{\lambda+2} Y_{\lambda \mu}(\hat{\vec{r}})
\end{aligned}
$$

$$
\begin{aligned}
\hat{M}_{\mathrm{com}}(E \lambda \mu)= & \frac{i}{2 c(2 \lambda+3)} \\
& \cdot \int d^{3} r \hat{\vec{j}}_{\mathrm{n} u c}(\vec{r}) \cdot \vec{\nabla}\left[r^{\lambda+2} Y_{\lambda \mu}(\hat{\vec{r}})\right]
\end{aligned}
$$
that both toroidal expressions (26) and (27) involve on $r^{\lambda+2} Y_{\lambda \mu}(\vec{r})$ thus manifesting the relation tween TM and CM. In (27), the second term precisely gives the CM operator 31 ].

In Eq. (16), the lowest-order $k$-terms from (23) and 24) exactly compensate each other and so we get

$$
\hat{M}_{\mathrm{vor}}(E \lambda \mu, k)=k\left[\hat{M}_{\mathrm{tor}}(E \lambda \mu)+\hat{M}_{\mathrm{com}}(E \lambda \mu)\right] .
$$

$$
\begin{aligned}
\hat{M}_{\mathrm{vor}}(E \lambda \mu) & =-\frac{i}{c(2 \lambda+3)} \sqrt{\frac{2 \lambda+1}{\lambda+1}} \cdot \int d^{3} r \hat{\vec{j}}_{\mathrm{n} u c}(\vec{r}) r^{\lambda+1} \vec{Y}_{\lambda \lambda+1 \mu}(\hat{\vec{r}}) \\
\hat{M}_{\mathrm{tor}}(E \lambda \mu) & =-\frac{i}{2 c} \sqrt{\frac{\lambda}{2 \lambda+1}} \int d^{3} r \hat{\vec{j}}_{\mathrm{n} u c}(\vec{r}) \cdot r^{\lambda+1}\left[\vec{Y}_{\lambda \lambda-1 \mu}(\hat{\vec{r}})+\sqrt{\frac{\lambda}{\lambda+1}} \frac{2}{2 \lambda+3} \vec{Y}_{\lambda \lambda+1 \mu}(\hat{\vec{r}})\right] \\
& =-\frac{1}{2 c} \sqrt{\frac{\lambda}{\lambda+1}} \frac{1}{2 \lambda+3} \int d^{3} r r^{\lambda+2} \vec{Y}_{\lambda \lambda \mu}(\hat{\vec{r}}) \cdot\left(\vec{\nabla} \times \hat{\vec{j}}_{\mathrm{n} u c}(\vec{r})\right) \\
\hat{M}_{\mathrm{com}}(E \lambda \mu) & =\frac{i}{2 c} \sqrt{\frac{\lambda}{2 \lambda+1}} \int d^{3} r \hat{\vec{j}}_{n u c}(\vec{r}) \cdot r^{\lambda+1}\left[\vec{Y}_{\lambda \lambda-1 \mu}(\hat{\vec{r}})-\sqrt{\frac{\lambda+1}{\lambda}} \frac{2}{2 \lambda+3} \vec{Y}_{\lambda \lambda+1 \mu}(\hat{\vec{r}})\right] \\
& =-\frac{i}{2 c} \frac{1}{2 \lambda+3} \int d^{3} r r^{\lambda+2} Y_{\lambda \mu}(\hat{\vec{r}})\left(\vec{\nabla} \cdot \hat{\vec{j}}_{\mathrm{n} u c}(\vec{r})\right) .
\end{aligned}
$$

Here, the TM and VM operators are the same as in (26) - (28) but are written in the forms convenient for the comparison with the VM operator. Besides these forms demonstrate the $\vec{\nabla} \times \hat{\vec{j}}_{\mathrm{n} u c}(\vec{r})$ and $\vec{\nabla} \cdot \hat{\vec{j}}_{\mathrm{n} u c}(\vec{r})$ origin of the 
TM and CM operators, respectively.

The expression for the VM operator (31) and the relation (30) between VM, TM, and CM operators represent the main formal results of the present paper.

Following (30), the operators $\hat{M}_{\mathrm{vor}}(E \lambda \mu), \hat{M}_{\mathrm{tor}}(E \lambda \mu)$, and $\hat{M}_{\text {com }}(E \lambda \mu)$ are of the same second order by $k$. They are given in (31)-(33) in the current-dependent form. Using the continuity equation (21), the current-dependent CM operator (33) is straightforwardly transformed to the familiar density-dependent CM operator 13.

$$
\hat{M}_{\text {com }}^{\prime}(E \lambda \mu)=\frac{1}{2(2 \lambda+3)} \int d^{3} r \hat{\rho}(\vec{r}) r^{\lambda+2} Y_{\lambda \mu}(\hat{\vec{r}})
$$

as

$$
\hat{M}_{\mathrm{com}}(E \lambda \mu)=-k \hat{M}_{\mathrm{com}}^{\prime}(E \lambda \mu) .
$$

Note that the relation (30) requires the compensation of the terms $\sim \vec{Y}_{\lambda \lambda-1 \mu}(\hat{\vec{r}})$ in the TM and CM operators. Thus a simultaneous use of these two operators is obligatory. The VM operator includes only $\vec{Y}_{\lambda \lambda+1 \mu}(\hat{\vec{r}})$ and so its matrix elements are determined by the current transition density $j_{\lambda \lambda+1}(r)$, as requested in [7]. It is easy to check that $\hat{M}_{\mathrm{vor}}(E \lambda \mu)$ gives the transition vorticity (11) and so reproduces the results [7].

The above formalism was derived for the case when the system is excited by the external electric field, i.e. for the excitation energy $\omega=\hbar c k>0$. The case of deexcitation is easily obtained by replacement $k \rightarrow-k$ in the continuity equations (5) and (21), k-dependent terms in (15), (27), (35), and equations of Appendix C. The sign of $\hat{M}_{S}(E \lambda \mu, k)$ and density-dependent $\hat{M}(E \lambda \mu)$ is changed as well.

\section{B. Dipole VM, TM, and CM operators}

The VM, TM, and CM are usually studied for the elec$\operatorname{tric} I^{\pi}=1^{-}$states [15]. Then the operators (31)-(33) are reduced to

$$
\begin{aligned}
& \hat{M}_{\mathrm{vor}}(E 1 \mu)=-\frac{i}{5 c} \sqrt{\frac{3}{2}} \int d^{3} r \hat{\vec{j}}_{\mathrm{n} u c}(\vec{r}) r^{2} \vec{Y}_{12 \mu}(\hat{\vec{r}}), \\
& \hat{M}_{\mathrm{tor}}(E 1 \mu)=-\frac{i}{2 \sqrt{3} c} \int d^{3} r \hat{\vec{j}}_{\mathrm{n} u c}(\vec{r}) \\
& \cdot\left[\frac{\sqrt{2}}{5} r^{2} \vec{Y}_{12 \mu}(\hat{\vec{r}})+\left(r^{2}-\delta_{T, 0}\left\langle r^{2}\right\rangle_{0}\right) \vec{Y}_{10 \mu}(\hat{\vec{r}})\right] \\
& \hat{M}_{\mathrm{com}}(E 1 \mu)=-\frac{i}{2 \sqrt{3} c} \int d^{3} r \hat{\vec{j}}_{\mathrm{n} u c}(\vec{r}) \\
& \cdot\left[\frac{2 \sqrt{2}}{5} r^{2} \vec{Y}_{12 \mu}(\hat{\vec{r}})-\left(r^{2}-\delta_{T, 0}\left\langle r^{2}\right\rangle_{0}\right) \vec{Y}_{10 \mu}(\hat{\vec{r}})\right] \\
& \hat{M}_{\mathrm{com}}^{\prime}(E 1 \mu)=\frac{1}{10} \int d^{3} r \hat{\rho}(\vec{r}) \\
& \quad \cdot\left[r^{3}-\delta_{T, 0} \frac{5}{3}\left\langle r^{2}\right\rangle_{0} r\right] Y_{1 \mu}(\hat{\vec{r}})
\end{aligned}
$$

where $\left\langle r^{2}\right\rangle_{0}=\int d^{3} r \rho_{0}(\vec{r}) r^{2} / A$ is the ground-state squared radius.

In (37)-(39), the terms $\sim \vec{Y}_{10 \mu}(\hat{\vec{r}}), Y_{1 \mu}(\hat{\vec{r}})$ include the center of mass corrections (c.m.c.) for $\mathrm{T}=0$ excitations [13]. In the TM and current-dependent CM operators, the c.m.c. have the same magnitude. For the VM, the c.m.c. is zero, see discussion in Appendix B

The expression in the square brackets of the TM operator (37) can be written as 13]

$$
\vec{\nabla} \times(\vec{r} \times \vec{\nabla})\left(r^{3}-\frac{5}{3}\left\langle r^{2}\right\rangle_{0} r\right) Y_{1 \mu}(\hat{\vec{r}}),
$$

which justifies a close relation between TM and CM.

\section{Discussion of VM, TM, and CM operators and vorticity criteria}

As shown above, the CM operator may be presented in the current-dependent (33) and density-dependent (34) forms. To the best of our knowledge, the former has not yet been used in the literature. Since the spin current $j_{m}$ is a curl of the magnetization, it does not contribute to the continuity equation and CM operator. Though the current-dependent form (33) of CM formally involves $j_{m}$, its contribution is annihilated by $\vec{\nabla} \cdot \hat{\vec{j}}_{\mathrm{n} u c}(\vec{r})$ or the corresponding combinations of vector spherical harmonics. So actually both forms, (33) and (34), of the CM operator do not depend on $j_{m}$.

Both CM operators, (33) and (34), are obtained through $\vec{\nabla} \cdot \hat{\vec{j}}_{\text {nuc }}(\vec{r})$, which suggests their vorticity-free character. This is confirmed by the form of the CM velocity which, following the prescription [46], reads

$$
\vec{v}_{\mathrm{com}}(\vec{r}) \propto \vec{\nabla} r^{\lambda+2} Y_{\lambda \mu}(\hat{\vec{r}})
$$

and so gives $\vec{\nabla} \times \vec{v}_{\mathrm{com}}(\vec{r})=0$.

The current-dependent CM operator (33) includes the $\vec{Y}_{\lambda \lambda+1 \mu}(\hat{\vec{r}})$ contribution which might be considered as an indicator of a vortical part. Indeed $\vec{Y}_{\lambda \lambda+1 \mu}(\hat{\vec{r}})$ leads to the current component $j_{\lambda \lambda+1}(r)$ which, following [7], is responsible for the vorticity. However, for the CM this is misleading. The velocity (41) can be straightforwardly cast into the form

$$
\begin{aligned}
\vec{v}_{\mathrm{com}}(\vec{r}) & \propto \sqrt{\frac{\lambda}{2 \lambda+1}} r^{\lambda+1} \\
& \cdot\left[\vec{Y}_{\lambda \lambda-1 \mu}(\hat{\vec{r}})-\sqrt{\frac{\lambda+1}{\lambda}} \frac{2}{2 \lambda+3} \vec{Y}_{\lambda \lambda+1 \mu}(\hat{\vec{r}})\right]
\end{aligned}
$$

whose curl is zero despite the $\vec{Y}_{\lambda \lambda+1 \mu}(\hat{\vec{r}})$ term. So, following the HD criterion (2), the appearance of a term $\vec{Y}_{\lambda \lambda+1 \mu}(\hat{\vec{r}})$ in the mode operator and current is not yet a definitive signature of the vorticity.

Altogether, we see apparent differences between two possible criteria for the vorticity: i) the HD condition 
(2) in terms of velocities, $\vec{\nabla} \times \vec{v}(\vec{r}) \neq 0$, and ii) the condition [7] in terms of transition current density components, $j_{\lambda \lambda+1}(r) \neq 0$. As shown above for CM example, a mode which is fully vorticity-free in the HD definition can have a substantial vorticity of the sort [7]. The difference between these criteria might be understood if we take into account that the vorticity density $w_{\lambda \lambda}(r) \propto j_{\lambda \lambda+1}(r)$ was derived in [7], first of all, as a quantity completely unconstrained by the charge conservation rather than a purely vortical value in the HD sense.

In fluid-dynamical and HD models [11, 28], the velocity fields are chosen in form of the relevant external fields exciting the proper modes. Following this practice, the TM velocity reads

$$
\begin{aligned}
\vec{v}_{\mathrm{tor}}(\vec{r}) & \propto \vec{\nabla} \times(\vec{r} \times \vec{\nabla}) r^{\lambda+2} Y_{\lambda \mu}(\hat{\vec{r}}) \\
& =i \sqrt{\frac{\lambda}{2 \lambda+1}}(\lambda+1)(2 \lambda+3) r^{\lambda+1} \\
& \cdot\left[\vec{Y}_{\lambda \lambda-1 \mu}(\hat{\vec{r}})+\sqrt{\frac{\lambda}{\lambda+1}} \frac{2}{2 \lambda+3} \vec{Y}_{\lambda \lambda+1 \mu}(\hat{\vec{r}})\right] .
\end{aligned}
$$

It is easy to check that $\vec{\nabla} \times \vec{v}_{\text {tor }} \propto r^{\lambda} \vec{Y}_{\lambda \lambda \mu}(\hat{\vec{r}})$ and so TM carries the HD vorticity.

The VM velocity constructed in the same manner is

$$
\vec{v}_{\mathrm{vor}}(\vec{r}) \propto r^{\lambda+1} \vec{Y}_{\lambda \lambda+1 \mu}(\hat{\vec{r}}) .
$$

It has the similar nonzero curl $\vec{\nabla} \times \vec{v}_{\text {vor }} \propto r^{\lambda} \vec{Y}_{\lambda \lambda \mu}(\hat{\vec{r}})$. So the VM has the vorticity of both HD and Ref. [7] sorts.

\section{CALCULATION SCHEME}

The calculations of the excitation modes were performed within the separable random-phase approximation (SRPA) model using the Skyrme energy functional [37, 38]. SRPA was earlier successfully applied to description of electric [37 41] and magnetic [43 45] GR in spherical and deformed nuclei. The approach was also used for the exploration of E1 strength near particle emission thresholds 42]. SRPA is fully self-consistent in the sense that both the static mean-field and factorized residual interaction are derived from the same Skyrme energy functional [49 51]. The functional, $\mathcal{E}_{\mathrm{Sk}}(\rho, \tau, \vec{J}, \vec{j}, \vec{s}, \vec{T})$, includes time-even (nucleon $\rho$, kinetic-energy $\tau$, spin-orbit $\vec{J}$ ) and time-odd (current $\vec{j}$, spin $\vec{s}$, vector kinetic-energy $\vec{T}$ ) densities. It also involves pairing (surface and volume), Coulomb (direct and exchange), and c.m.c. terms 37, 38, 43. The Galilean invariance of the functional is maintained in SRPA. The tensor spin-orbit contribution is involved through the squared spin-orbit densities $\vec{J}^{2}$. All the functional terms are kept in the mean field and residual interaction.

The SRPA expands the RPA residual interaction selfconsistently into a sum of separable terms, which dramatically reduces the computational effort while keeping the accuracy of the full (non-separable) RPA [37, 38].
This makes SRPA extremely useful for systematic calculations and tasks with very large configuration space, e.g. for description of GR in heavy deformed nuclei.

For GR studies, the computational expense can be even more reduced by a direct evaluation of the strength function, thus avoiding the solution of RPA equations for a large multitude of individual states. The SRPA strength function works with the Lorentz weight and has very simple form [37, 38]. In the present study, the strength function for electric dipole modes reads

$$
S_{\alpha}(E 1 ; \omega)=(2 \pi)^{2} \sum_{\mu=0, \pm 1} \sum_{\nu}\left|\left\langle\Psi_{\nu}\left|\hat{M}_{\alpha}(E 1 \mu)\right| \Psi_{0}\right\rangle\right|^{2} \zeta\left(\omega-\omega_{\nu}\right)
$$

where

$$
\zeta\left(\omega-\omega_{\nu}\right)=\frac{1}{2 \pi} \frac{\Delta}{\left(\omega-\omega_{\nu}\right)^{2}+\frac{\Delta^{2}}{4}}
$$

is the Lorentz weight with the smoothing width $\Delta$ and $\hat{M}_{\alpha}(E 1 \mu)$ is the electric dipole transition operator whose type is determined by the index $\alpha=$ $\left\{\right.$ vor, tor, com, com'\}. Further, $\Psi_{0}$ is the ground state, $\nu$ runs over the RPA spectrum with eigen-frequencies $\omega_{\nu}$ and eigen-states $\left|\Psi_{\nu}\right\rangle$. The Lorentz smoothing uses a width $\Delta=1 \mathrm{MeV}$ to simulate broadening effects beyond RPA (escape widths and coupling to complex configurations). The explicit form of the strength function (46), which does not directly involve the RPA solutions, is given elsewhere [37, 38].

In general, the VM, TM, and CM appear at many different multipolarities $\lambda \mu$. Here we explore them in the $I^{\pi}=1^{-}$excitations of the doubly-magic spherical nucleus ${ }^{208} \mathrm{~Pb}$. In this nucleus the pairing is absent. The calculations use the Skyrme parameterization SLy6 [52], which provides a satisfactory description of the $\mathrm{E} 1(\mathrm{~T}=1)$ GR in heavy nuclei [41]. For this parameterization, the tensor spin-orbit contribution is omitted.

The calculations employ a cylindrical coordinate-space grid with the mesh size $0.7 \mathrm{fm}$. A large single-particle basis is used. The particle-hole $1^{-}$pairs extend up to $\sim 65 \mathrm{MeV}$ and, for $\mathrm{E} 1(\mathrm{~T}=1)$ excitations, the energyweighted sum rule with the isovector effective mass is exhausted by $\sim 95 \%$ 41.

The calculations involve both convection $j_{c}$ and magnetization (spin) $j_{m}$ parts of the nuclear current, see Appendix $\mathrm{C}$ for more details. For $j_{m}$, the isoscalar $g_{s}^{n, p}(T=0)=\left(g_{s}^{p}+g_{s}^{n}\right) / 2=0.88 \varsigma$ and isovector $g_{s}^{n, p}(T=1)=\left(g_{s}^{n}-g_{s}^{p}\right) / 2=-4.70 \varsigma$ gyromagnetic factors are used, where $g_{s}^{p}=5.58 \varsigma$ and $g_{s}^{n}=-3.82 \varsigma$ are bare proton and neutron $g$-factors and $\varsigma=0.7$ is a quenching parameter taking approximately into account the meson degrees of freedom [53]. It is easy to see that $\left|g_{s}^{n, p}(T=1)\right| \gg\left|g_{s}^{n, p}(T=0)\right|$ and so the main effect of the spin nuclear current is expected for $\mathrm{T}=1$ modes.

The proton and neutron $(q=n, p)$ RPA velocity fields are determined in cylindrical coordinates $(z, \rho)$ as

$$
\vec{v}_{\nu}^{q}(z, \rho)=\frac{\delta \vec{j}_{\nu q}(z, \rho)}{\rho_{0}^{q}(z, \rho)}
$$


where

$$
\delta \vec{j}_{\nu q}(z, \rho)=\sum_{i j \in q}\left\langle i j\left|\hat{\vec{j}}_{n u c}^{q}\right| 0\right\rangle\left(c_{i j}^{\nu q-}-c_{i j}^{\nu q+}\right)
$$

is the current transition density for the RPA $\nu$-state with the normalized forward and backward particle-hole (1ph) amplitudes $c_{i j}^{\nu q-}$ and $c_{i j}^{\nu q+}$. Further, $\rho_{0}^{q}(z, \rho)$ is the proton/neutron ground state density.

The spurious c.m. admixtures are avoided by using the prescriptions from the Appendix B The SRPA equations and some important points, e.g. a choice of the generating operators for the separable expansion, are sketched in the Appendix D

\section{RESULTS AND DISCUSSION}

Results of the calculations for the nucleus ${ }^{208} \mathrm{~Pb}$ are presented in Figs. 119,

In Figure 1 the VM, TM, and CM strengths in $\mathrm{T}=0$ and 1 channels are compared. The strengths are computed for the transition operators (36)-(38). For the CM, the current-dependent operator $\hat{M}_{\text {com }}$ is used. Unlike its familiar density-dependent counterpart $\hat{M}_{\text {com }}^{\prime}$, it has the same dimension as the VM and TM operators and so is more suitable for the comparison of the modes. All the modes are calculated with total nuclear current $j_{n u c}$.

Figure 1 shows that all the modes have basically two broad branches, a low-energy branch (LEB) at 5-20 MeV and a high-energy branch (HEB) at 25-40 MeV. The VM is well presented in both branches while TM and CM are mainly localized in LEB and HEB, respectively. Such a double-branch structure was found for the TM and CM in most of the previous theoretical studies [15]. It is most probably related to E1 transitions with $\Delta N=1$ and 3 where $N$ is the principle shell number.

The double-branch structure of the $\mathrm{E} 1(\mathrm{~T}=0)$ strength was confirmed by various experiments [14, 18, 20 25], mainly in $\left(\alpha, \alpha^{\prime}\right)$ scattering at small angles. The recent results of this reaction 24, 25] are depicted in Fig. 1a). The reaction is considered as a common tool for measurements of the dipole $\mathrm{CM}(\mathrm{T}=0)$ [53].

As seen from the panel a) of Fig. 1, our results do not reproduce the experimental energies and widths of the LEB and HEB. Neither of the mode centroids coincides with the experimental peak energies. Note that this is a common shortcoming of almost all theoretical studies performed within various theoretical approaches [15]. Namely, the theory i) underestimates by $1-2 \mathrm{MeV}$ the TM-dominated LEB and overestimates by about $4 \mathrm{MeV}$ the CM-dominated HEB, ii) yields a much broader TM distribution and a too narrow $\mathrm{CM}$ one. The reason of the discrepancies is still unclear. Perhaps, this is partly caused by neglecting the coupling with complex configurations. Figure 1shows that the VM and TM strengths are of the same order of magnitude in the LEB left flank for $\mathrm{T}=0$ and in the whole LEB for $\mathrm{T}=1$ (the difference

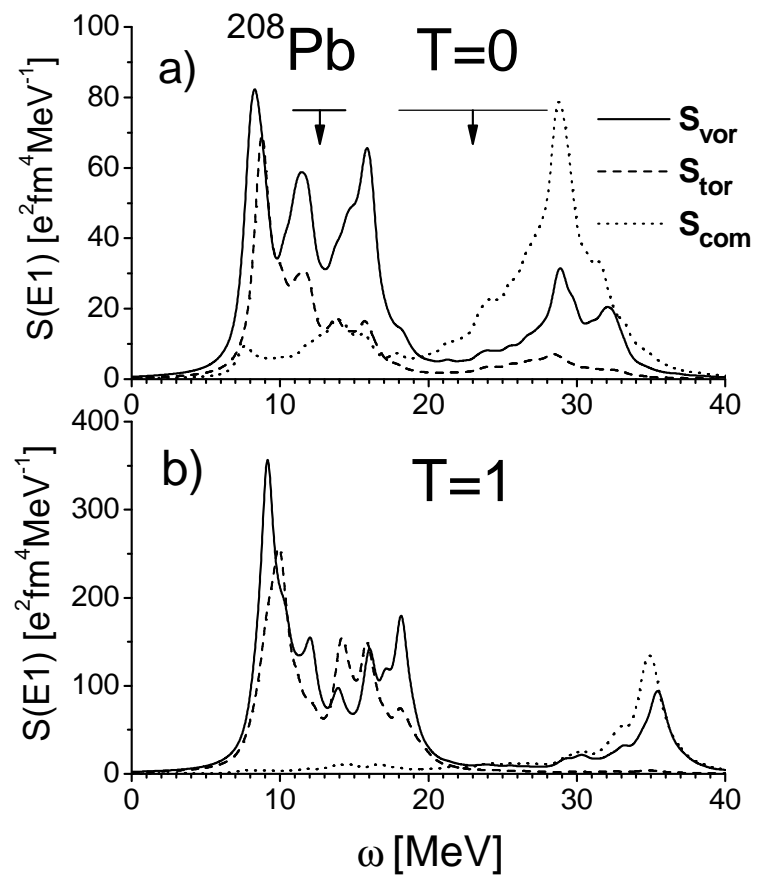

FIG. 1: Isoscalar $(\mathrm{T}=0)$ and isovector $(\mathrm{T}=1)$ vortical, toroidal, and compression dipole modes in ${ }^{208} \mathrm{~Pb}$, calculated with the SLy6 parameterization. The total nuclear current $j_{n u c}$ is used. The CM is computed with the operator $\hat{M}_{\text {com }}$ from (38). The lines with the arrows indicate widths and energy centroids of the low-energy and high-energy branches of isoscalar E1 excitations observed in $\left(\alpha, \alpha^{\prime}\right)$ reaction [24, 25].

between $\mathrm{T}=0$ and 1 cases is explained below in the discussion of Figs. 2 and 3). Perhaps, in these regions the TM is mainly vortical. We also see that VM dominates at the right LEB flank and is significant in HEB where the TM contribution is weak. The CM strictly dominates in HEB and has a noticeable tail in LEB at $\mathrm{T}=0$. The latter is because of the coupling between TM and CM [13, 31]. Obviously, the difference between VM, on the one hand, and TM and CM, on the other hand, is mainly explained by the terms $\sim \vec{Y}_{10 \mu}(\hat{\vec{r}})$ which are absent in $\hat{M}_{\text {vor }}$ but active in $\hat{M}_{\text {tor }}$ and $\hat{M}_{\text {com }}$. Just because of these terms, Fig. 1 cannot be used for a direct check of the relation (30). For the same reason, the similarity of VM and CM strengths in the HEB cannot be considered as a signature of the CM vorticity because VM and CM represent essentially different kinds of the motion, vortical versus irrotational. Instead, this rather means that both kinds of motion are presented by E1 $\Delta N=3$ transitions.

The obtained results suggest that the $\operatorname{VM}(\mathrm{T}=0)$ may be hopefully disentangled from other modes in $\left(\alpha, \alpha^{\prime}\right)$ at the excitation energy $\sim 16 \mathrm{MeV}$, where the $\operatorname{VM}(\mathrm{T}=0)$ strictly dominates. For $\left(e, e^{\prime}\right)$, the pygmy region $7-10$ $\mathrm{MeV}$ seems to be most promising to observe $\mathrm{VM}$ and TM. In this region a dominant contribution $j_{12}(r)$ of the nuclear current is expected.

In Figure 2, the isoscalar VM and TM strengths, calculated with the complete $j_{n u c}=j_{c}+j_{m}$, convection $j_{c}$, 


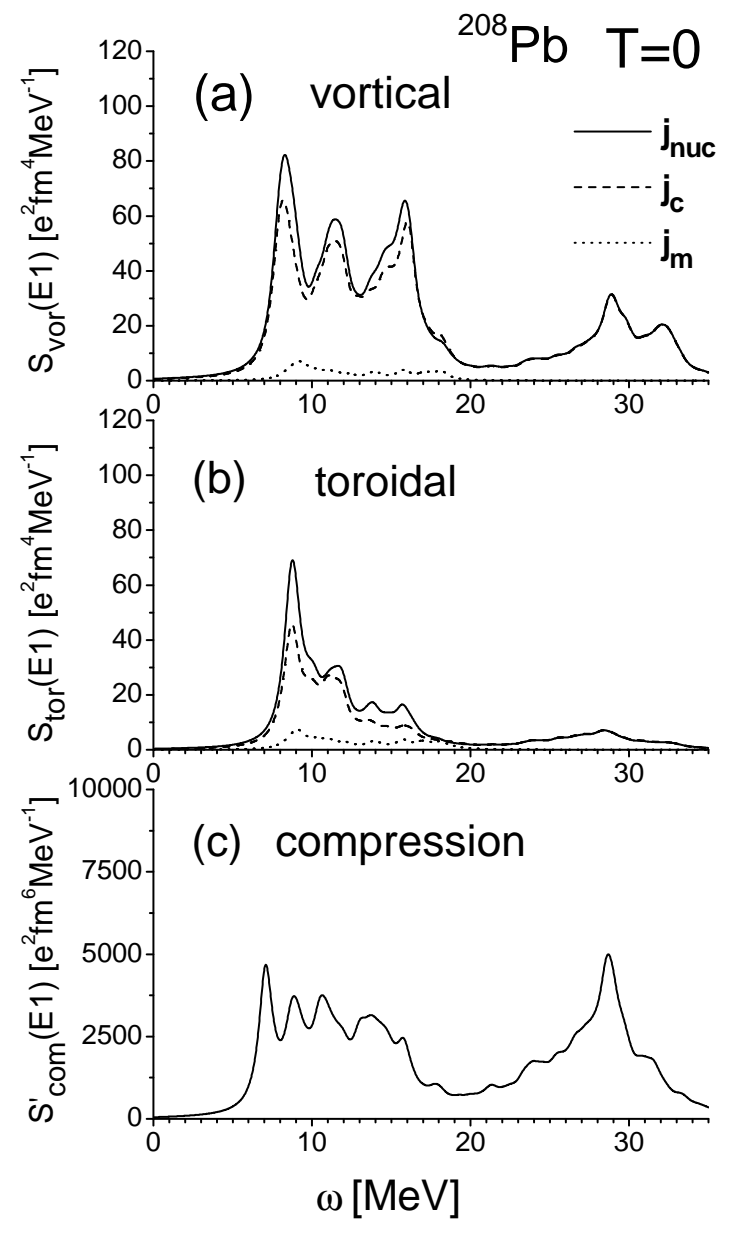

FIG. 2: Calculated isoscalar $(\mathrm{T}=0)$ vortical, toroidal, and compression dipole modes in ${ }^{208} \mathrm{~Pb}$. For the VM and TM, the strengths with the total $j_{n u c}$, convection $j_{c}$, and magnetization $j_{m}$ current contributions to the transition operators are shown.

and magnetization $j_{m}$ nuclear currents, are compared. It is seen that the $j_{m}$ contribution is weak and so the $\mathrm{T}=0$ VM and TM are mainly of the convection nature. This is especially the case for the HEB where the $j_{m}$ contribution, being mainly of low-energy spin-flip character, vanishes at all. The weakness of the $j_{m}$ weight in $\mathrm{T}=0$ channel is naturally explained by the low values of the gyromagnetic factors $g_{s}^{q}(T=0)$, as mentioned in Sec. IV] Fig. 22 also exhibits the CM. Unlike Fig. [1, here the familiar density-dependent operator $\hat{M}_{\mathrm{com}}^{\prime}$ is used. Following (35), $\hat{M}_{\text {com }}^{\prime}$ is less energy-weighted than $\hat{M}_{\text {com }}$ and so gives a more comparable CM strength in LEB and HEB. As discussed above, the CM is determined by $\nabla \cdot \vec{j}_{n u c}$ and so is purely irrotational. It has no any contribution from $j_{m}$ and thus is fully of convective.

In Figure 3, the VM, TM, and CM are shown in the $\mathrm{T}=1$ channel. As compared to the previous $\mathrm{T}=0$ case, we see dramatic changes in the magnitude and composition of VM and TM. In the LEB, these modes become stronger and dominated by the $j_{m}$ contribution. The rea-

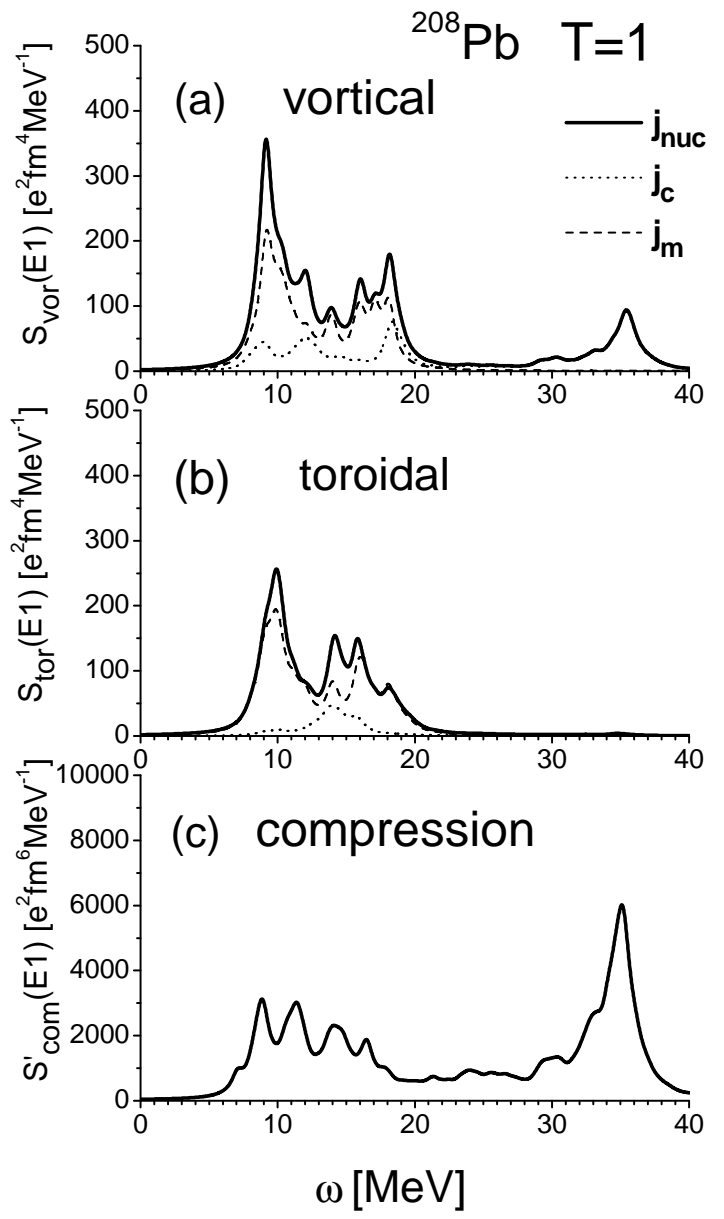

FIG. 3: The same as in Fig. 2 but for the $\mathrm{T}=1$ modes.

son of the changes is obvious. The isovector spin factors, $g_{s}^{n, p}(T=1)=-4.70 \varsigma$, are much larger than the isoscalar ones, $g_{s}^{n, p}(T=0)=0.88 \varsigma$. So, the $\mathrm{T}=1$ spin contribution grows about $\left(g_{s}^{n, p}(T=1) / g_{s}^{n, p}(T=0)\right)^{2} \sim 29$ times. It becomes dominant and significantly increases the total VM and TM strengths. Note that this effect does not concern the HEB which remains purely convective. Besides, the $j_{m}$ effect is zero for the CM.

The next point to be considered is collectivity of the modes. To this end, Figs. 4 and 5 compare RPA and unperturbed particle-hole (1ph) strengths. It is seen that the RPA residual interaction noticeably down-shifts the strength for $\mathrm{T}=0$ and up-shifts it for $\mathrm{T}=1$. The maximal collective shifts (defined as a difference between RPA and 1ph peaks) take place in the $\mathrm{CM}$, where they reach 1-2 $\mathrm{MeV}$ in LEB and 2-4 MeV in HEB. The HEB shift is comparable to that of the $\mathrm{E} 1(\mathrm{~T}=1)$ giant dipole resonance (GDR) exhibited in Fig. 6 and so is indeed very large. This indicates that HEB modes, VM and CM, are collective. The LEB modes, for exception of a few high peaks, are less collective. The LEB almost coincides with the region of the unperturbed $1 p h$ dipole strength depicted in Fig. 6] and so for the LEB the single-particle aspect is also important. These observations are confirmed by in- 


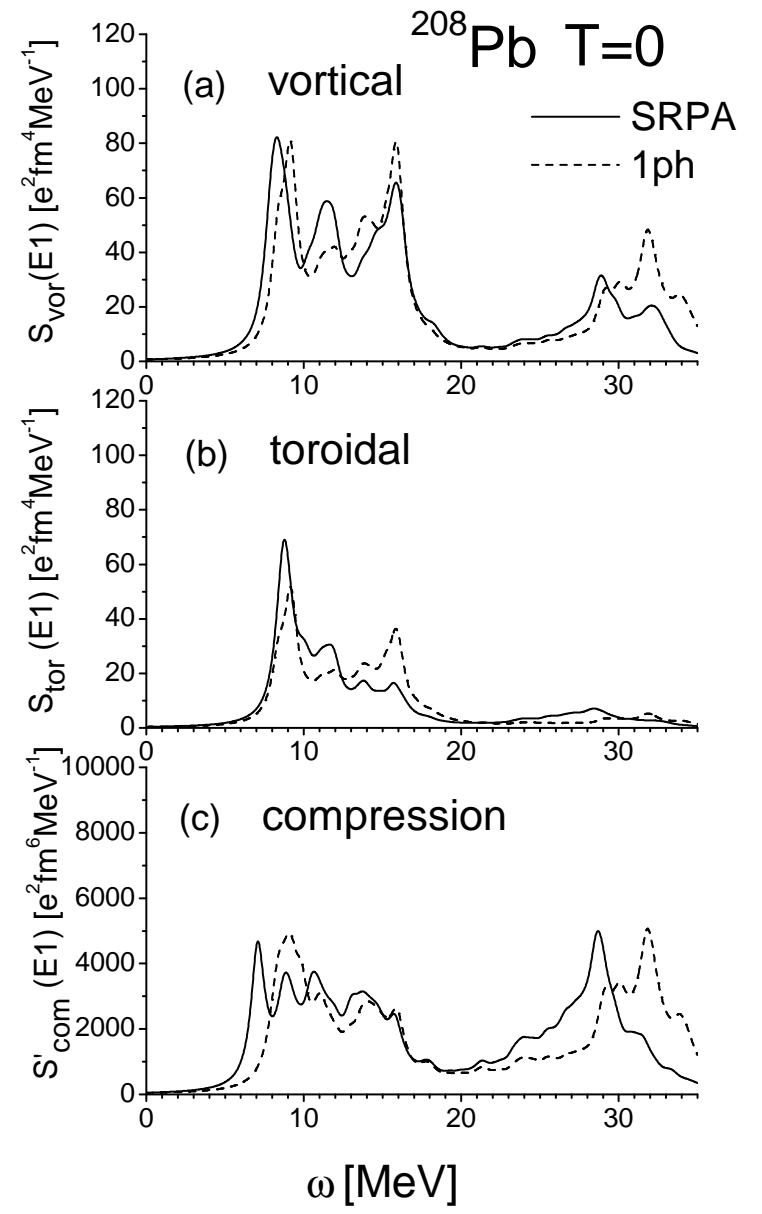

FIG. 4: Comparison of SRPA and 1ph strengths for the $\mathrm{T}=0$ modes. For VM and TM, the strengths are computed with the total nuclear current $j_{\text {nuc }}$.

spection of the detailed structure of the RPA states and agree with the previous studies [15] for the high-energy CM and low-energy TM.

The next figures take a closer view at the detailed structures of a few most collective LEB modes. In Figs. 7.9, the VM, TM, and CM neutron and proton velocity fields (48) for particular $\mathrm{T}=0$ and $\mathrm{T}=1 \mathrm{RPA}(\mu=0)$ states are shown. The states in the region $6-10 \mathrm{MeV}$ with the maximal VM, TM, and CM responses are considered: 8.3 MeV (VM), 8.7 MeV (TM), 7.1 MeV (CM) for $\mathrm{T}=0$ and $9.1 \mathrm{MeV}$ (VM), 9.8 MeV (TM), 8.8 MeV (CM) for $\mathrm{T}=1$. These states are easily recognized in Figs. 2 2 [5 as highest peaks at the left side of the LEB. The states combine collectivity and single-particle effects: their structure is a coherent superposition of many $1 p h$ components with maximal contributions $20-40 \%$. The velocities are plotted in cylindrical coordinates $(z, \rho)$ and, for simplicity, only the quadrant $(z>0, \rho>0)$ is presented.

Figures 749 show that velocity fields are rather involved, indicating a complex structure of the RPA states. The clear imprints of the vortex motion are seen as local curls. Sometimes, the fields well reproduce a typical

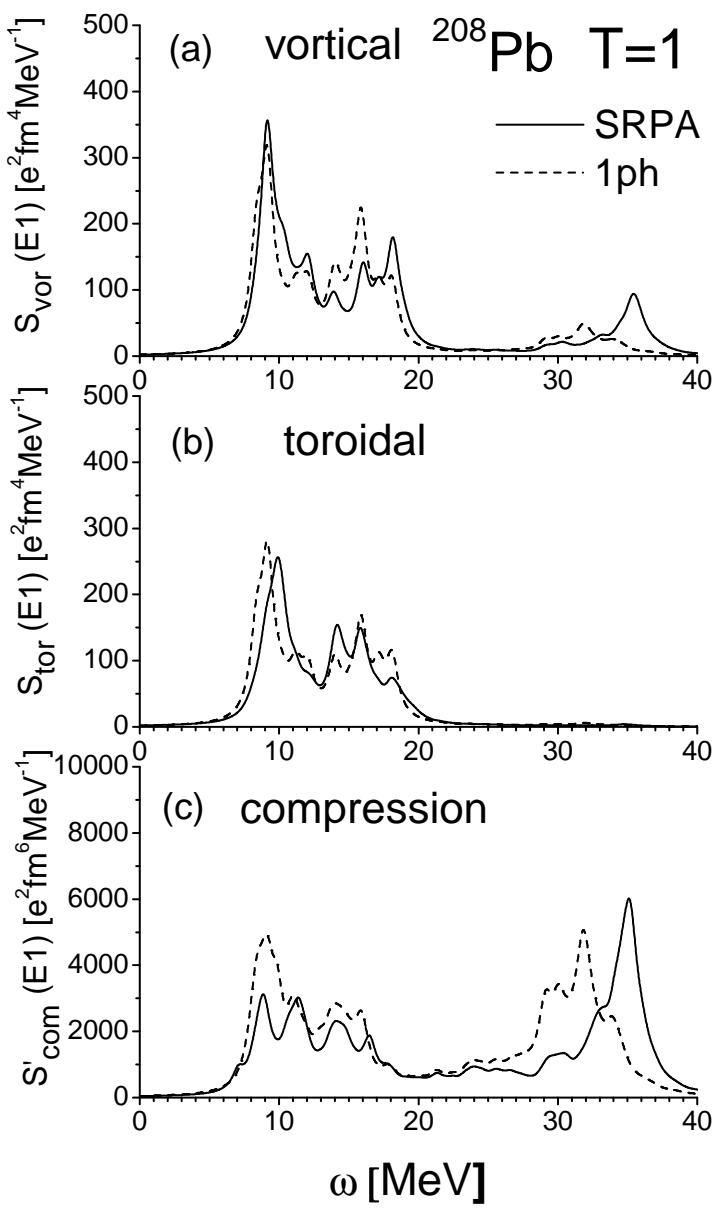

FIG. 5: The same as in Fig. 4 but for the $\mathrm{T}=1$ modes.

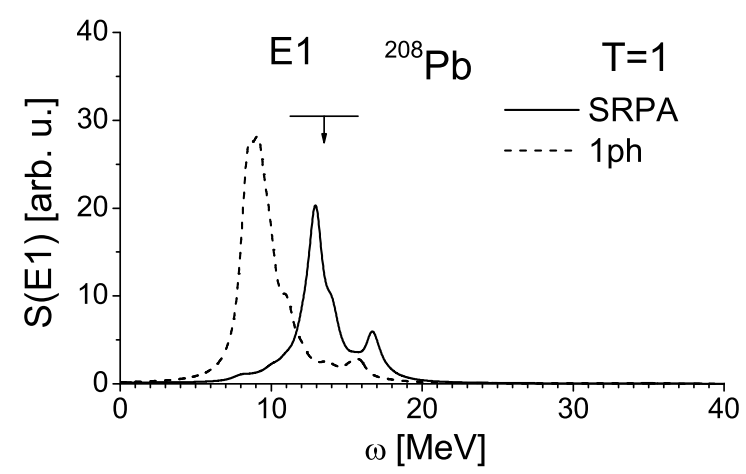

FIG. 6: SRPA and 1ph strengths for the E1(T=1) GDR. Like for other modes, the strength functions are plotted without the energy weight. The experimental width and energy 54 are shown by the horizontal line and arrow, respectively.

toroidal picture, see e.g. the $\mathrm{T}=0$ proton velocities for VM and CM in panels b) of Figs. 7 and 9 The curls for the CM may be explained by its strong coupling to TM in LEB. In Fig. 9 $\mathrm{a}$ ), the strong dipole component is also seen. However, in most of the panels, a large impact of the single-particle motion, e.g. of the nodal structure of the leading $1 \mathrm{ph}$ components, distorts the familiar collec- 


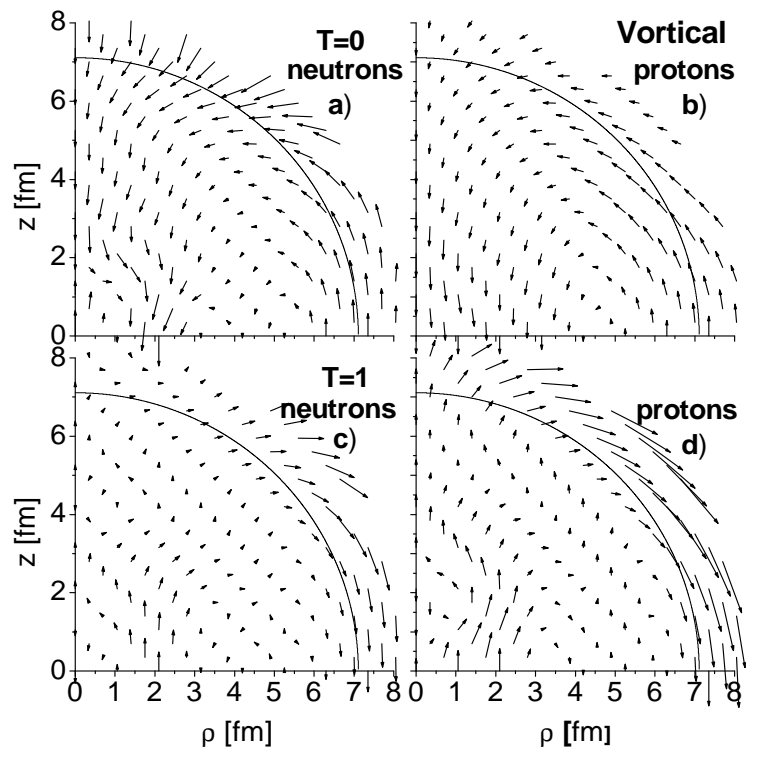

FIG. 7: a)-b): Neutron and proton vortical velocity fields $\vec{v}_{\nu}^{q}(z, \rho)$ for the state $\omega_{\nu}=8.3 \mathrm{MeV}$ with a maximal $\mathrm{T}=0$ vortical response. c)-d): The same for the state $\omega_{\nu}=9.1$ $\mathrm{MeV}$ with a maximal $\mathrm{T}=1$ vortical response. For a better view, the velocities are amplified by the factors 50 (a-b) and 20 (c-d).

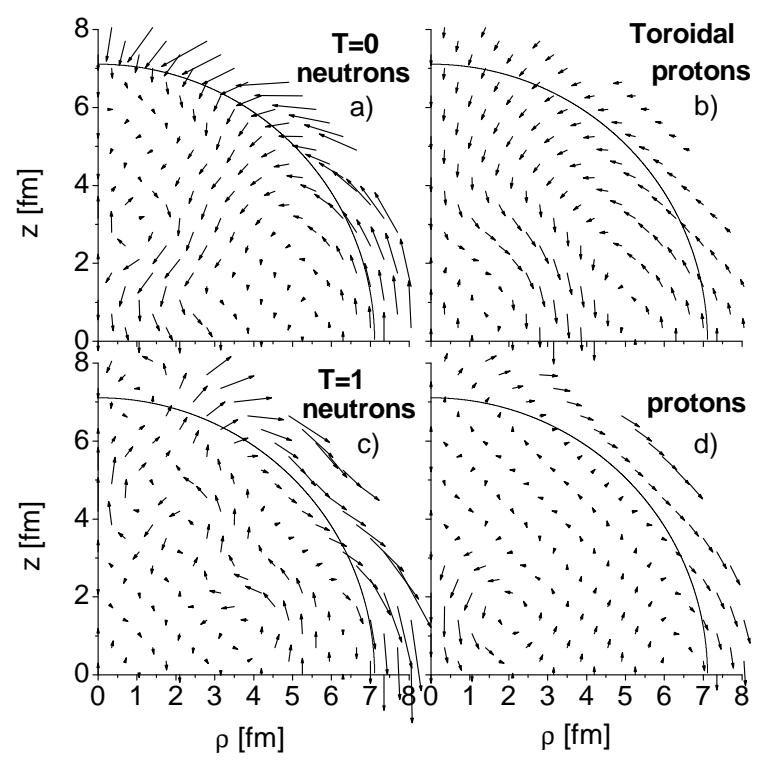

FIG. 8: The same as in Fig. 7 but for the states $\omega_{\nu}=$ $8.7 \mathrm{MeV}(\mathrm{T}=0)$ and $\omega_{\nu}=9.8 \mathrm{MeV}(\mathrm{T}=1)$ with a maximal toroidal strength.

tive TM and CM flows and considerably complicates the picture.

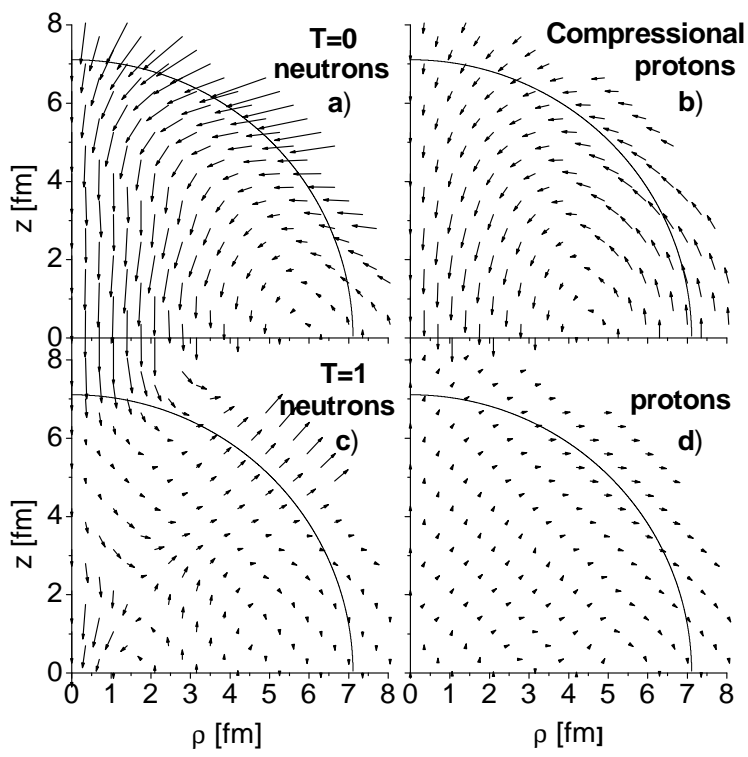

FIG. 9: The same as in Fig. 7 but for the states $\omega_{\nu}=$ $7.1 \mathrm{MeV}(\mathrm{T}=0)$ and $\omega_{\nu}=8.8 \mathrm{MeV}(\mathrm{T}=1)$ with a maximal compression strength in the low-energy branch.

\section{CONCLUSIONS}

The nuclear vorticity and relevant vortical, toroidal, and compression modes (VM, TM, and CM) were explored on general formal grounds. The operators of all three modes were derived as second-order terms in the long-wavelength expansion of the electrical multipole operator and its subsidiary counterpart built following the concept 7]. For the first time, the vortical operator was constructed and related to its toroidal and compression counterparts. The detailed comparison of the modes and their operators was done. It was explicitly shown that, while VM (CM) is vortical (irrotational) by construction, the TM is of a mixed character. The vorticity criteria from HD and arguments based on the decoupling to the charge conservation [7] were inspected. It was shown that the latter deviates from the HD definition and may lead occasionally to misleading conclusions as, e.g., a vorticity of the CM.

The electric dipole VM, TM, and CM were computed and investigated in ${ }^{208} \mathrm{~Pb}$ within the self-consistent Skyrme random-phase-approximation (RPA) approach. Unlike most of the previous studies, both convection and magnetization (spin) parts of the nuclear current were taken into account and both isoscalar $(T=0)$ and isovector $(\mathrm{T}=1)$ channels of the modes were analyzed. It was shown that VM and CM have low-energy and high-energy branches while TM mainly appears in the low-energy branch. The CM strictly dominates in a high energy branch. In the $\mathrm{T}=0$ channel, the $\mathrm{VM}$ and $\mathrm{TM}$ are almost completely determined by the nuclear convection current while in the $\mathrm{T}=1$ channel, their low-energy 
branches are strictly dominated by the spin current. This strong isospin effect is straightforwardly explained by low (high) values of $\mathrm{T}=0(\mathrm{~T}=1)$ spin $\mathrm{g}$-factors, which drastically changes the ratio between the convective and spin contributions of the current. The effect cannot appear in the CM since this irrotational mode has vanishing spin contribution.

The collectivity was found strong for the high-energy VM and CM and rather weak for the low-energy VM, TM, and CM. In the latter case, the velocity fields of the modes are rather involved. This is partly a consequence of the complex structure of the RPA states mixing the collective with detailed $1 p h$ contributions. This holds in particular for the vorticity which seems to be of both single-particle and collective origin.

The VM, TM, and CM were shown to be closely related and, at the same time, display considerable differences in their detailed strength distribution. These modes seem to represent one family with complementing aspects. It would be interesting to analyze the results of our study more deeply and use them to disentangle the modes in the $\left(e, e^{\prime}\right)$ and hadron reactions. This is in our next plans.

\section{Acknowledgments}

The work was partly supported by the DFG RE322/12-1, Heisenberg-Landau (Germany - BLTP JINR), and Votruba - Blokhintsev (Czech Republic - BLTP JINR) grants. W.K. and P.-G.R. are grateful for the BMBF support under contracts 06 DD 9052D and 06 ER 9063. Being a part of the research plan MSM 0021620859 (Ministry of Education of the Czech Republic) this work was also funded by Czech grant agency (grant No. 202/09/0084). The work of P.V. was partly supported by the Academy of Finland and the University of Jyväskylä within the FIDIPRO program.

\section{Appendix A: Removal of the charge conservation constraint}

In Sec. III A, the vortical operator $\hat{M}_{\mathrm{vor}}(E \lambda \mu, k)$, completely unconstrained by the continuity equation, is built from the $\hat{M}(E \lambda \mu, k)$ operator by replacing the curl of the nuclear current $\vec{\nabla} \times \hat{\vec{j}}_{\text {nuc }}(\vec{r})$ by the vortical quantity

$$
\hat{\vec{\omega}}_{\lambda}=\left[\vec{\nabla} \times \hat{\vec{j}}_{\mathrm{n} u c}(\vec{r})\right]_{\lambda}-\frac{i}{\lambda} k c[\vec{\nabla} \hat{\rho}(\vec{r}) \times \vec{r}]_{\lambda}
$$

where the terms $[\ldots]_{\lambda}$ assume $\lambda$-components of the multipole expansion of the values inside the brackets. Below we present arguments which motivate (A1) and compare it with the prescription [7]. For the simplicity, we consider only the convection current and neglect isospin.

\section{Simple arguments}

Using the HD definition of the velocity field (3), we may write the truly vortical quantity

$$
\rho_{0}(\vec{r}) \vec{\nabla} \times \vec{v}_{\nu}(\vec{r})=\vec{\nabla} \times \delta \vec{j}_{\nu}(\vec{r})-\vec{\nabla} \rho_{0}(\vec{r}) \times \hat{\vec{v}}_{\nu}(\vec{r}) .
$$

Except for the second velocity-dependent term, the r.h.s. of (A2) reminds the operator construction (A1) and thus may be used for justification of $\hat{\vec{\omega}}_{\lambda}$ as a vortical quantity.

To make (A2) closer to (A1), it is worth to express $\vec{r}$ in terms of the (global) velocity operator

$$
\hat{\overrightarrow{\mathrm{v}}}=\dot{\overrightarrow{\vec{r}}}=\frac{i}{\hbar}[\hat{H}, \hat{\vec{r}}]=i k c \hat{\vec{r}}
$$

This suggests the replacement

$$
\hat{\vec{v}} \rightarrow i k c \hat{\vec{r}}
$$

in (A2) and thus casts A2 to a form similar to A1 (up to the multiplier $1 / \lambda)$. Note, however, that the velocities in (A2) and A3 are not the same. The replacement of the velocity field $\vec{v}_{\nu}(\vec{r})$ by a global velocity becomes strictly valid only in the sum-rule limit when all multipole strength is concentrated in one collective state. It remains probably an acceptable step for distributed spectra which often gather around a strongly collective mode.

\section{Correspondence of the recipes}

It is also worth to relate the recipe (10) for the transition densities [7] with our recipe (15) and its analog (A1) for the operators. As was mentioned in Sec. III A, both recipes serve to build the vortical quantities, though by different ways. While (10) excludes from the current the $j_{\lambda \lambda-1}(r)$ terms, the recipe (15) leads to exact compensation of the lowest-order $k$-terms in the vortical operator.

One may show that the recipes (10) and (15) actually correspond each other. This may be done by treating (15) in terms of the transition densities (6) and currents (17). Using the relation between the density and current expansion multipoles

$$
\begin{aligned}
k c \rho_{\lambda}(r)= & -\sqrt{\frac{\lambda}{2 \lambda+1}}\left\langle\frac{d}{d r}-\frac{\lambda-1}{r}\right\rangle j_{\lambda \lambda-1}(r) \\
& +\sqrt{\frac{\lambda+1}{2 \lambda+1}}\left\langle\frac{d}{d r}+\frac{\lambda+2}{r}\right\rangle j_{\lambda \lambda+1}(r),
\end{aligned}
$$

one may show that (15) indeed leads to the unconstrained vortical transition density (10). In this case, the second r.h.s. term of Eq. (A1) has the form

$$
i k c\left[\vec{\nabla} \delta \rho_{f i}(\vec{r}) \times \vec{r}\right]=\sum_{\lambda \mu} a_{\lambda \mu}^{f i} S_{\lambda \lambda}(r) \vec{Y}_{\lambda \lambda \mu}(\hat{\vec{r}})
$$

with the multipoles

$$
S_{\lambda \lambda}(r)=\sqrt{\lambda(\lambda+1)} k c \rho_{\lambda}(r) .
$$

Being scaled by $1 / \lambda$, these multipoles coincide with the expansion coefficients of $\delta \vec{S}_{f i}(\vec{r})$ in (9). 


\section{Appendix B: Extraction of spurious admixtures}

The isoscalar electric dipole VM, TM, and CM may have spurious admixtures related to the center of mass motion of the nucleus. There are various methods to derive the center of mass corrections (c.m.c.), see e.g. [4, 15, 55 57]. Most of the methods neglect the velocity, spin-, and spin-orbit-dependent terms in the nuclear interaction and assume a closure relation where all excitation is contained in one single collective state. Hence the methods are approximate. Nevertheless, being simple and convenient, they are widely used in the SkyrmeHartree-Fock calculations [15]. Below we use the method [56] to derive the c.m.c. for isoscalar TM and CM. For the isoscalar VM, the c.m.c. is shown to be zero.

Let's consider a system with the Hamiltonian $\hat{H}=$ $\hat{T}+\hat{V}$ whose interaction $\hat{V}$ does not depend on spin and velocities. Then, in the isoscalar $(\mathrm{T}=0)$ case, for any one-body external field $\hat{F}=\sum_{i}^{A} f\left(\overrightarrow{r_{i}}\right)$, where $f\left(\overrightarrow{r_{i}}\right)$ is an arbitrary function of nucleon coordinates, one may write the sum rules [46]

$$
\begin{aligned}
\sum_{\nu} \omega_{\nu}\langle 0|\hat{\rho}(\vec{r})| \nu\rangle\langle\nu|\hat{F}| 0\rangle & =-\frac{1}{2 m} \vec{\nabla} \cdot\left[\rho_{0}(\vec{r}) \vec{\nabla} f(\vec{r})\right](\mathrm{B} 1) \\
\sum_{\nu}\langle 0|\hat{\vec{j}}(\vec{r})| \nu\rangle\langle\nu|\hat{F}| 0\rangle & =\frac{1}{2 m i} \rho_{0}(\vec{r}) \vec{\nabla} f(\vec{r})
\end{aligned}
$$

for the isoscalar transition density $\langle 0|\hat{\rho}(\vec{r})| \nu\rangle$ and current $\langle 0|\hat{\vec{j}}(\vec{r})| \nu\rangle$. The sum runs through the full set of the excitation eigenstates $|\nu\rangle\left(\hat{H}|\nu\rangle=\omega_{\nu}|\nu\rangle\right)$ with the eigenvalues $\omega_{\nu}$. Further, $\rho_{0}(\vec{r})=\left\langle 0\left|\sum_{i}^{A} \delta\left(\vec{r}-\vec{r}_{i}\right)\right| 0\right\rangle$ is the nuclear ground state density, and $m$ is the nucleon mass.

If the external field mainly excites one single collective state $\bar{\nu}$, then only the term $(\nu=\bar{\nu})$ survives in (B1)- (B2) and the corresponding transition density and current are uniquely specified through $\rho_{0}(\vec{r})$ and $f(\vec{r})$ [46]:

$$
\begin{aligned}
\langle 0|\hat{\rho}(\vec{r})| \bar{\nu}\rangle & =-\frac{1}{2 m} \frac{1}{\omega_{\bar{\nu}}\langle\bar{\nu}|\hat{F}| 0\rangle} \vec{\nabla} \cdot\left[\rho_{0}(\vec{r}) \vec{\nabla} f(\vec{r})\right] \\
\langle 0|\hat{\vec{j}}(\vec{r})| \bar{\nu}\rangle & =\frac{1}{2 m i} \frac{1}{\langle\bar{\nu}|\hat{F}| 0\rangle} \rho_{0}(\vec{r}) \vec{\nabla} f(\vec{r})
\end{aligned}
$$

Hence we get simple recipes for the transition densities and currents,

$$
\begin{aligned}
\delta \rho(\vec{r}) & \propto \vec{\nabla} \cdot\left[\rho_{0}(\vec{r}) \vec{\nabla} f(\vec{r})\right], \\
\delta \vec{j}(\vec{r}) & \propto \rho_{0}(\vec{r}) \vec{\nabla} f(\vec{r}) \propto \rho_{0}(\vec{r}) \vec{v}(\vec{r}),
\end{aligned}
$$

to be used in further c.m.c. derivation. Here (B6) actually defines an irrotational flow with the velocity $\vec{v}(\vec{r}) \sim \vec{\nabla} f(\vec{r})$.

A change of the expectation value $\langle\hat{O}\rangle$ of any one-body operator $\hat{O}=\sum_{i}^{A} o\left(\vec{r}_{i}\right)$, caused by an external field $\hat{F}$, is

$$
\begin{aligned}
\delta\langle\hat{O}\rangle & =\int d^{3} r \delta \rho(\vec{r}) o(\vec{r}) \\
& \propto \int d^{3} r o(\vec{r}) \vec{\nabla} \cdot\left[\rho_{0}(\vec{r}) \vec{\nabla} f(\vec{r})\right]= \\
& =-\int d^{3} r \rho_{0}(\vec{r}) \vec{\nabla} f(\vec{r}) \cdot \vec{\nabla} o(\vec{r}) \\
& \propto-\int d^{3} r \delta \vec{j}(\vec{r}) \cdot \vec{\nabla} o(\vec{r})
\end{aligned}
$$

where we use the relations (B5)-(B6). Both $\delta \rho(\vec{r})$ and $\delta \vec{j}(\vec{r})$ may be applied to determine $\delta\langle\hat{O}\rangle$. These cases are suitable for the modes determined by the density-dependent and current-dependent operators, respectively. Note that Eq. (B10) with $\delta \vec{j}(\vec{r})$ is general and may be derived and used by itself, regardlessly to the formalism (B1)- (B9) and character of the flow. The quantity $\delta \vec{j}(\vec{r})$ is then determined by Eq. (3) and, unlike (B6), the velocity $\vec{v}(\vec{r})$ of the flow can be not only irrotational but also vortical or mixed. Altogether, the relations (B7)-B10 may be reduced to an expression

$$
\delta\langle\hat{O}\rangle \approx \int d^{3} r \rho_{0}(\vec{r}) \vec{v}(\vec{r}) \cdot \vec{\nabla} o(\vec{r})
$$

with $\vec{v}(\vec{r})$ covering both irrotational (B6) and more general cases.

If $\langle\hat{O}\rangle$ is invariant with respect to the field $\hat{F}$, then the requirement $\delta\langle\hat{O}\rangle=0$ is kept and (B11) gives

$$
\int d^{3} r \rho_{0}(\vec{r}) \vec{v}(\vec{r}) \cdot \vec{\nabla} o(\vec{r})=0
$$

For

$$
o(\vec{r})=r Y_{1 \mu}^{*}(\hat{\vec{r}}),
$$

the operator $\hat{O}=\sum_{i}^{A} o\left(\vec{r}_{i}\right)$ describes the c.m. coordinate and (B12) means that this coordinate is not affected by the field $\hat{F}$. By using

$$
\vec{\nabla} o(\vec{r})=\vec{\nabla} r Y_{1 \mu}^{*}(\hat{\vec{r}})=\sqrt{3} \vec{Y}_{10 \mu}^{*}(\hat{\vec{r}}),
$$

the conditions (B12) is cast into

$$
\int d^{3} r \rho_{0}(\vec{r}) \vec{v}(\vec{r}) \cdot \vec{Y}_{10 \mu}^{*}(\hat{\vec{r}})=0 .
$$

The condition (B15) may be applied to the densitydependent CM operator $\hat{M}_{\text {com }}^{\prime}(E 1 \mu)$ by imposing the irrotational velocity

$$
\vec{v}_{\text {com }}(\vec{r})=\vec{\nabla} f_{\text {com }}(\vec{r})=\vec{\nabla} Y_{1 \mu}(\hat{\vec{r}})\left(r^{3}-\eta r\right)
$$

where the second term is the actual c.m.c. with the coefficient $\eta$ to be determined from (B15). Then, assuming spherical nuclei $\left(\rho_{0}(\vec{r})=\rho_{0}(r)\right)$ and using

$$
\int d^{3} r \vec{Y}_{\lambda l \mu}^{*}(\hat{\vec{r}}) \cdot \vec{Y}_{\lambda^{\prime} l^{\prime} \mu^{\prime}}(\hat{\vec{r}})=\delta_{l l^{\prime}} \delta_{\lambda \lambda^{\prime}} \delta_{\mu \mu^{\prime}},
$$


we get

$$
\eta=\frac{5}{3}\left\langle r^{2}\right\rangle_{0}
$$

with

$$
\left\langle r^{2}\right\rangle_{0}=\frac{\int_{0}^{\infty} r^{4} \rho_{0}(r) d r}{\int_{0}^{\infty} r^{2} \rho_{0}(r) d r}
$$

and finally the expression (39) for $\hat{M}_{\text {com }}^{\prime}(E 1 \mu)$.

For the current-dependent VM, TM, and CM operators (36) - (38), we put to (B15) the velocities

$$
\begin{aligned}
\vec{v}_{\mathrm{v} o r}(\vec{r}) & =r^{2} \vec{Y}_{12 \mu}(\hat{\vec{r}})-\eta \vec{Y}_{10 \mu}(\hat{\vec{r}}) \\
\vec{v}_{\mathrm{tor}}(\vec{r}) & =\frac{\sqrt{2}}{5} r^{2} \vec{Y}_{12 \mu}(\hat{\vec{r}})+\vec{Y}_{10 \mu}(\hat{\vec{r}})\left(r^{2}-\eta\right) \\
\vec{v}_{\mathrm{com}}(\vec{r}) & =\frac{\sqrt{2}}{5} r^{2} \vec{Y}_{12 \mu}(\hat{\vec{r}})-\vec{Y}_{10 \mu}(\hat{\vec{r}})\left(r^{2}-\eta\right)
\end{aligned}
$$

where the second terms with $\eta$ are the relevant c.m.c.. For exception of $\vec{v}_{\text {com }}(\vec{r})$, these velocities are not reduced to the gradient form. Actually, they are taken in the form of the external fields involved in the operators (36)- (38) and generating the corresponding modes. Such presentation is in accordance with the self-consistent treatment of nuclear excitations [1], which is done here in terms of small variations $\delta \vec{j}(\vec{r})$ of the nuclear current.

By using (B20)-(B22), the requirement (B15) gives

$$
\eta=0
$$

for VM and

$$
\eta=\left\langle r^{2}\right\rangle_{0}
$$

for TM and CM. Thus we get the corrected expression (37)-(38) for $\hat{M}_{\text {tor }}(E 1 \mu)$ and $\hat{M}_{\text {com }}(E 1 \mu)$. Note that vector harmonics $\vec{Y}_{12 \mu}(\hat{\vec{r}})$ related to the vorticity do not contribute to the c.m.c.. This reflects the physical fact that vorticity, being a curl flow, must be fully decoupled from the c.m. translation motion. Hence the c.m.c. is zero for the VM. On the other hand, the TM is not completely vortical and so its c.m.c. does not vanish.

Note that the above c.m.c. are approximate. Indeed, the calculations give for the VM, TM, and CM responses two broad structures, which actually do not meet the sum-rule condition of excitation of a single collective state. Besides, the prescription [46] uses the commutator $[\hat{H}, \hat{\rho}]$ where $\hat{H}$ is assumed not to include the terms with velocity-, spin-, and spin-orbit dependence. However, the effect of spin-dependent terms in the commutator is obviously zero. The momentum (velocity)-dependent interaction does not matter for the Galilean-invariant Skyrme functional (the most common case) but may be important if this invariance is violated. The spin-orbit interaction may affect the c.m.c..

\section{Appendix C: Nuclear density and current operators}

The density operator reads

$$
\hat{\rho}(\vec{r})=\sum_{q=n, p} e_{\mathrm{eff}}^{q} \sum_{k \in q}\left(\delta\left(\vec{r}-\vec{r}_{k}\right)\right.
$$

where $e_{\text {eff }}^{q}$ are proton and neutron effective charges.

The operator of the full nuclear current consists of the convective and magnetic (spin) parts 48]

$$
\hat{\vec{j}}_{\mathrm{n} u c}(\vec{r})=\hat{\vec{j}}_{c}(\vec{r})+\hat{\vec{j}}_{m}(\vec{r})=\frac{e \hbar}{m} \sum_{q=n, p}\left(\hat{\vec{j}}_{c}^{q}(\vec{r})+\hat{\vec{j}}_{m}^{q}(\vec{r})\right)
$$

where

$$
\begin{aligned}
& \hat{\vec{j}}_{c}^{q}(\vec{r})=-i e_{\mathrm{eff}}^{q} \sum_{k \in q}\left(\delta\left(\vec{r}-\vec{r}_{k}\right) \vec{\nabla}_{k}+\vec{\nabla}_{k} \delta\left(\vec{r}-\vec{r}_{k}\right)\right) \\
& \hat{\vec{j}}_{m}^{q}(\vec{r})=\frac{g_{s}^{q}}{2} \sum_{k \in q} \vec{\nabla} \times \hat{\vec{s}}_{q k} \delta\left(\vec{r}-\vec{r}_{k}\right)
\end{aligned}
$$

and $\hat{\vec{s}}_{q}$ is the spin operator, $\mu_{N}$ is the nuclear magneton, $g_{s}^{q}$ is the spin g-factor, $k$ numerates the nucleons.

The $\mathrm{T}=0$ modes use the values

$$
e_{\mathrm{eff}}^{n}=e_{\mathrm{eff}}^{p}=1, \quad g_{s}^{n, p}(T=0)=\frac{1}{2}\left(g_{s}^{n}+g_{s}^{p}\right)
$$

while the $\mathrm{T}=1$ modes employ

$$
e_{\mathrm{eff}}^{n}=-e_{\mathrm{eff}}^{p}=-1, \quad g_{s}^{n, p}(T=1)=\frac{1}{2}\left(g_{s}^{n}-g_{s}^{p}\right) .
$$

\section{Appendix D: SRPA equations and generator operators}

The SRPA Hamiltonian is self-consistently derived 37 , 38. from the functional

$$
\mathcal{E}=\mathcal{E}_{\text {kin }}+\mathcal{E}_{\mathrm{Sk}}+\mathcal{E}_{\text {pair }}+\mathcal{E}_{\mathrm{Coul}}
$$

involving kinetic-energy, Skyrme, pairing and Coulomb terms. The Skyrme functional $\mathcal{E}_{\mathrm{Sk}}(\rho, \tau, \vec{J}, \vec{j}, \vec{s}, \vec{T})$ depends on time-even (nucleon $\rho$, kinetic-energy $\tau$, spinorbit $\vec{J}$ ) and time-odd (current $\vec{j}$, spin $\vec{s}$, vector kineticenergy $\vec{T}$ ) densities. The Hamiltonian reads [37, 38]

$$
\hat{H}=\hat{h}_{\mathrm{HFB}}+\hat{V}_{\mathrm{res}}
$$

where $\hat{h}_{\mathrm{HFB}}$ is the HFB mean field

$$
\hat{h}_{\mathrm{HFB}}=\int d^{3} r \sum_{\alpha_{+}}\left[\frac{\delta E}{\delta J_{\alpha_{+}}(\vec{r})}\right] \hat{J}_{\alpha_{+}}
$$

and $\hat{V}_{\text {res }}$ is the separable residual interaction

$$
\hat{V}_{\text {res }}=\frac{1}{2} \sum_{k, k^{\prime}=1}^{K}\left(\kappa_{k k^{\prime}} \hat{X}_{k} \hat{X}_{k^{\prime}}+\eta_{k k^{\prime}} \hat{Y}_{k} \hat{Y}_{k^{\prime}}\right)
$$


with one-body operators

$$
\begin{aligned}
& \hat{X}_{k}=i \int d^{3} r d^{3} r^{\prime} \sum_{\alpha_{+}, \alpha_{+}^{\prime}} \frac{\delta^{2} \mathcal{E}}{\delta J_{\alpha_{+}} \delta J_{\alpha_{+}^{\prime}}}\left\langle\left[\hat{P}_{k}, \hat{J}_{\alpha_{+}}\right]\right\rangle \hat{J}_{\alpha_{+}^{\prime}}, \\
& \hat{Y}_{k}=i \iint d^{3} r d^{3} r^{\prime} \sum_{\alpha_{-}, \alpha_{-}^{\prime}} \frac{\delta^{2} \mathcal{E}}{\delta J_{\alpha_{-}} \delta J_{\alpha_{-}^{\prime}}}\left\langle\left[\hat{Q}_{k}, \hat{J}_{\alpha_{-}}\right]\right\rangle \hat{J}_{\alpha_{-}^{\prime}},
\end{aligned}
$$

and inverse strength matrices

$$
\kappa_{k k^{\prime}}^{-1}=-i\left\langle\left[\hat{P}_{k}, \hat{X}_{k^{\prime}}\right]\right\rangle, \quad \eta_{k k^{\prime}}^{-1}=-i\left\langle\left[\hat{Q}_{k}, \hat{Y}_{k^{\prime}}\right]\right\rangle .
$$

Here $\alpha_{+}$and $\alpha_{-}$enumerate time-even $J_{\alpha_{+}}$and time-odd $J_{\alpha_{-}}$densities, respectively; $\hat{J}_{\alpha_{ \pm}}$are the density operators; $\hat{Q}_{k}$ and $\hat{P}_{k}=i\left[\hat{H}, \hat{Q}_{k}\right]$ are time-even and time-odd hermitian generator operators. The operators of the residual interaction $\hat{X}_{k}$ and $\hat{Y}_{k}$ are time-even and time-odd by construction, respectively.

The single-particle Hamiltonian $\hat{h}_{\mathrm{HFB}}$ is determined by the first functional derivatives of the initial functional (D1) while operators $\hat{X}_{k}$ and $\hat{Y}_{k}$ are driven by the second functional derivatives of the same functional. The residual interaction includes all the possible terms arising from (D1). Hence the model is fully self-consistent. The number $K$ of separable terms in (D4) is determined by the number of the generator (input) operators $\hat{Q}_{k}$. Usually we have $K=3-5$. This results in a low rank of the RPA matrix and so in an efficient calculation scheme.

The SRPA formalism itself does not prescribe the form of the generators $\hat{Q}_{k}$ and $\hat{P}_{k}$. At the same time, their choice is important for a fast converge of the factorized residual interaction $\hat{V}_{\text {res }}$ to the true one with a minimal number of separable terms. The set of the generating operators is introduced so as to initiate in the nucleus all the relevenat kinds of motion for the considered modes. For time-even modes, the initial generators $\hat{Q}_{k}$ are chosen first and then their time-odd counterparts are determined from $\hat{P}_{k}=i\left[\hat{H}, \hat{Q}_{k}\right]$. Instead for time-odd modes, the initial generators $\hat{P}_{k}$ are inserted and then their time-even counterparts $\hat{Q}_{k}=i\left[\hat{H}, \hat{P}_{k}\right]$ are determined. The generators may be arbitrarily and separately scaled, which does not influence the results. The coupling of the modes (e.g. of electric and magnetic ones in deformed nuclei) may request both time-even and time-odd generators in the set. The optimal sets of the generators were developed for $\mathrm{E} 1(\mathrm{~T}=1)$ [37, 38] and spin-flip M1 [43 45] GR.

Here we use the minimal sets of the generators suitable for the description of VM, TM, and CM. The generators cover the main parts of the corresponding operators and take into account the coupling between the modes. For the VM, they are

$$
\begin{aligned}
\hat{P}_{1} & =\int d^{3} r \hat{\vec{j}}_{\mathrm{c}}(\vec{r}) r^{2} \vec{Y}_{12 \mu}(\hat{\vec{r}}), \\
\hat{P}_{2} & =\int d^{3} r \hat{\vec{j}}_{\mathrm{m}}(\vec{r}) r^{2} \vec{Y}_{12 \mu}(\hat{\vec{r}}), \\
\hat{Q}_{3} & =\int d^{3} r \rho(\vec{r})\left[r^{3}-\frac{5}{3}\left\langle r^{2}\right\rangle_{0} r\right] Y_{1 \mu}(\hat{\vec{r}}),
\end{aligned}
$$

i.e. cover the time-odd parts of the vortical operator with the convection and magnetization currents as well as the time-even compression operator with the c.m.c. (to prevent generation of the spurious motion).

For the TM, the generators read

$$
\begin{aligned}
\hat{P}_{1} & =\int d^{3} r \hat{\vec{j}}_{\mathrm{c}}(\vec{r})\left[r^{2} \frac{\sqrt{2}}{5} \vec{Y}_{12 \mu}(\hat{\vec{r}})+\vec{Y}_{10 \mu}(\hat{\vec{r}})\left(r^{2}-\left\langle r^{2}\right\rangle_{0}\right)\right], \\
\hat{P}_{2} & =\int d^{3} r \hat{\vec{j}}_{\mathrm{m}}(\vec{r})\left[r^{2} \frac{\sqrt{2}}{5} \vec{Y}_{12 \mu}(\hat{\vec{r}})+\vec{Y}_{10 \mu}(\hat{\vec{r}})\left(r^{2}-\left\langle r^{2}\right\rangle_{0}\right)\right], \\
\hat{Q}_{3} & =\int d^{3} r \rho(\vec{r})\left[r^{3}-\frac{5}{3}\left\langle r^{2}\right\rangle_{0} r\right] Y_{1 \mu}(\hat{\vec{r}}),
\end{aligned}
$$

i.e. cover the time-odd parts of the toroidal operator with the convection and magnetization currents as well as the time-even compression operator. The TM needs the c.m.c. and so now this correction is included to all the generators.

Finally the set for the CM includes the compression operator $\hat{Q}_{3}$ itself and the convective toroidal generator $\hat{P}_{1}$. The generators with the magnetic current $\hat{\vec{j}}_{\mathrm{m}}(\vec{r})$ are not involved since their effect on the $\mathrm{CM}$ is zero.
[1] A. Bohr and B. R. Mottelson, Nuclear Structure Vol. 2 (Benjamin, New York, 1974).

[2] T. de Forest and J.D. Walecka, Adv. Phys. 15, 57 (1966).

[3] J. Heisenberg, Adv. Nucl. Phys. 12, 61 (1981).

[4] P. Ring and P. Schuck, Nuclear Many Body Problem, (Springer-Verlag N.Y.-Hedelberg-Berlin, 1980).

[5] G.T. Bertsch, Nucl. Phys. A249, 253 (1975).

[6] F.E. Seer, T.S. Dumitrescu, T. Suzuki, C.H. Dasso, Nucl. Phys. A404, 359 (1983).

[7] D.G. Raventhall, J. Wambach, Nucl. Phys. A475, 468 (1987).

[8] E.C. Caparelli and E.J.V. de Passos, J. Phys. G: Nucl. Part. Phys. 25, 537 (1999).
[9] L. D. Landau and E. M. Lifshitz, Course of Theoretical Physics: Hydrodynamics Vol. 6, (ButterworthHeinemann, Oxford, 1987)

[10] J. Heisenberg, J. Lichtenstadt, C.N. Papanicolas, and J.S. McCarthy, Phys. Rev. C25, 2292 (1982).

[11] S.Misicu, Phys.Rev. C73, 024301 (2006).

[12] V.M. Dubovik and A.A. Cheshkov, Sov. J. Part. Nucl. 5, 318 (1975); V.M. Dubovik and L.A. Tosunyan, ibid, 14, 504 (1983).

[13] S.F. Semenko, Sov. J. Nucl. Phys. 34, 356 (1981).

[14] H.L. Clark, Y.W. Lui, and D.H. Youngblood, Phys.Rev. C63, 031301(R) (2001).

[15] N. Paar, D. Vretenar, E. Khan, and G. Colo, Rep. Prog. 
Phys. 70, 691 (2007).

[16] M.N. Harakeh et al, Phys. Rev. Lett. 38, 676 (1977).

[17] D.H. Youngblood et al, Phys. Rev. Lett. 39, 1188 (1977).

[18] H.P. Morsch, M. Rogge, P. Turek, and C. Mayer-Boricke, Phys. Rev. Lett. 45, 337 (1980).

[19] S. Stringari, Phys. Lett. B108, 232 (1982).

[20] H.P. Morsch et al, Phys. Rev. C28, 1947 (1983).

[21] G.S. Adams et al, Phys. Rev. C33, 2054 (1986).

[22] B.F. Davis et al, Phys. Rev. Lett. 79, 609 (1997).

[23] D.H. Youngblood et al, Phys.Rev. C69, 054312 (2004).

[24] M. Uchida et al, Phys. Lett. B 557, 12 (2003).

[25] M. Uchida et al, Phys. Rev. C 69, 051301(R) (2004).

[26] N. Ryezayeva et al, Phys. Rev. Lett. 89, 272502 (2002).

[27] A. Richter, Nucl. Phys. A731, 59 (2004).

[28] S.I. Bastrukov, S. Misicu, and A. Sushkov, Nucl. Phys. A562, 191 (1993).

[29] E.B. Balbutsev and I.N. Mikhailov, J.Phys.G 14, 545 (1988).

[30] E.B. Balbutsev, I.V. Molodtsova, and A.V. Unzhakova, Europhys. Lett. 26, 499 (1994).

[31] J. Kvasil, N. Lo Iudice, Ch. Stoyanov, and P. Alexa, J. Phys. G: Nucl. Part. Phys. 29, 753 (2003).

[32] D. Vretenar, A. Wandelt, and P. Ring, Phys. Lett. B487, 334 (2000).

[33] D. Vretenar, N. Paar, P. Ring, and T. Niksic, Phys. Rev. C65, 021301(R) (2002).

[34] G. Colo, N. Van Giai, P. Bortignon, and M.R. Quaglia, Phys.Lett. B485, 362 (2000).

[35] P. Papakonstantinou, J. Wambach, E. Mavrommatis, and V.Yu. Ponomarev, Phys. Lett. B 604, 157 (2004).

[36] E. Wuest, V. Mosel, J. Kunz, and A. Schuh, Nucl. Phys. A406, 285 (1987).

[37] V.O. Nesterenko, J. Kvasil, and P.-G. Reinhard, Phys. Rev. C66, 044307 (2002).

[38] V.O. Nesterenko, W. Kleinig, J. Kvasil, P. Vesely, P.G. Reinhard, and D.S. Dolci, Phys. Rev. C74, 064306 (2006).

[39] V.O. Nesterenko, W. Kleinig, J. Kvasil, P. Vesely, and
P.-G. Reinhard, Int. J. Mod. Phys. E16, 624 (2007).

[40] V.O. Nesterenko, W. Kleinig, J. Kvasil, P. Vesely, and P.-G. Reinhard, Int. J. Mod. Phys. E17, 89, (2008).

[41] W. Kleinig, V.O. Nesterenko, J. Kvasil, P.-G. Reinhard, and P. Vesely, Phys. Rev. C78, 044313 (2008).

[42] J. Kvasil, P. Vesely, V.O. Nesterenko W. Kleinig, P.-G. Reinhard, and S. Frauendorf, Int. J. Mod. Phys. E18, 975 (2009).

[43] P. Vesely, J. Kvasil, V.O. Nesterenko, W. Kleinig, P.G. Reinhard, and V.Yu. Ponomarev, Phys. Rev. C80, 031302(R) (2009).

[44] V.O. Nesterenko, J. Kvasil, P. Vesely, W. Kleinig, P.-G. Reinhard, and V.Yu. Pomomarev, J. Phys. G: Nucl. Part. Phys. 37, 064034 (2010).

[45] V.O. Nesterenko, J. Kvasil, P. Vesely, W. Kleinig, and P.-G. Reinhard, Int. J. Mod. Phys. E19, 558 (2010).

[46] T. Suzuki and D.J. Rowe, Nucl. Phys. A286, 307 (1977).

[47] D.A. Varshalovich, A.N. Moskalev, V.K. Khersonskii, Quantum Theory of Angular Momentum (World Scientific, Singapore, 1976).

[48] A. Bohr and B. R. Mottelson, Nuclear Structure Vol. 1 (Benjamin, New York, 1969).

[49] T.H.R. Skyrme, Phil. Mag. 1, 1043 (1956).

[50] D. Vauterin, D.M. Brink, Phys. Rev. C5, 626 (1972).

[51] Y.M. Engel, D.M. Brink, K. Goeke, S.J. Krieger, and D. Vauterin, Nucl. Phys. A249, 215 (1975).

[52] E. Chabanat, P. Bonche, P. Haensel, J. Meyer, and R. Schaeffer, Nucl. Phys. A635, 231 (1998).

[53] M.N. Harakeh and A. van der Woude, Giant Resonances (Clarendon Press, Oxford, 2001).

[54] A.V. Varlamov, V.V. Varlamov, D.S. Rudenko, and M.E. Stepanov, Atlas of Giant Dipole Resonances, INDC(NDS)-394, 1999.

[55] E. Lipparini and S. Stringari, Phys. Rep. 175, 103 (1989).

[56] N. Van Giai and H. Sagawa, Nucl. Phys. A371, 1 (1981).

[57] K.W. Schmid and P.-G. Reinhard, Nucl. Phys. A530, 283 (1991). 